\title{
Longest cycles in 3-connected hypergraphs and bipartite graphs
}

\author{
Alexandr Kostochka* $\quad$ Mikhail Lavrov ${ }^{\dagger} \quad$ Ruth Luo $^{\ddagger} \quad$ Dara Zirlin ${ }^{\S}$
}

April 20, 2020

\begin{abstract}
In the language of hypergraphs, our main result is a Dirac-type bound: we prove that every 3-connected hypergraph $\mathcal{H}$ with $\delta(\mathcal{H}) \geq \max \left\{|V(\mathcal{H})|, \frac{|E(\mathcal{H})|+10}{4}\right\}$ has a hamiltonian Berge cycle.

This is sharp and refines a conjecture by Jackson from 1981 (in the language of bipartite graphs). Our proofs are in the language of bipartite graphs, since the incidence graph of each hypergraph is bipartite.
\end{abstract}

Mathematics Subject Classification: 05C35, 05C38, 05C65, $05 \mathrm{D} 05$.

Keywords: Longest cycles, degree conditions, pancyclic hypergraphs.

\section{Introduction}

\subsection{Long cycles in bipartite graphs}

For positive integers $n, m$, and $\delta$ with $\delta \leq m$, let $\mathcal{G}(n, m, \delta)$ denote the set of all bipartite graphs with a partition $(X, Y)$ such that $|X|=n \geq 2,|Y|=m$ and for every $x \in X, d(x) \geq \delta$. In 1981, Jackson [3] proved that if $\delta \geq \max \left\{n, \frac{m+2}{2}\right\}$, then every graph $G \in \mathcal{G}(n, m, \delta)$ contains a cycle of length $2 n$, i.e., a cycle that covers $X$. This result is sharp. Jackson also conjectured that if $G \in \mathcal{G}(n, m, \delta)$ is 2-connected, then the upper bound on $m$ can be weakened.

Conjecture 1.1 (Jackson [3, 4]). Let $m, n, \delta$ be integers. If $\delta \geq \max \left\{n, \frac{m+5}{3}\right\}$, then every 2connected graph $G \in \mathcal{G}(n, m, \delta)$ contains a cycle of length $2 n$.

Recently, the conjecture was proved in [7]. The restriction $\delta \geq \frac{m+5}{3}$ cannot be weakened because of the following example.

Construction 1.2. Let $n_{1} \geq n_{2} \geq n_{3} \geq 1$ be such that $n_{1}+n_{2}+n_{3}=n$. Let $G_{3}\left(n_{1}, n_{2}, n_{3} ; \delta\right) \in$ $\mathcal{G}(n, 3 \delta-4, \delta)$ be the bipartite graph obtained from $K_{\delta-2, n_{1}} \cup K_{\delta-2, n_{2}} \cup K_{\delta-2, n_{3}}$ by adding two vertices $a$ and $b$ that are both adjacent to every vertex in the parts of size $n_{1}, n_{2}$, and $n_{3}$. Then a longest cycle in $G_{3}\left(n_{1}, n_{2}, n_{3} ; \delta\right)$ has length $2\left(n_{1}+n_{2}\right) \leq 2(n-1)$.

The goal of this paper is to find a best lower bound on $\delta$ guaranteeing the existence of a $2 n$-cycle in a graph $G \in \mathcal{G}(n, m, \delta)$ if $G$ is not only 2-connected, but 3-connected. The following simple extension of Construction 1.2 shows that the bound could not be larger than $\frac{m+10}{4}$.

\footnotetext{
*University of Illinois at Urbana-Champaign, Urbana, IL 61801 and Sobolev Institute of Mathematics, Novosibirsk 630090, Russia. E-mail: kostochk@math.uiuc.edu. Research is supported in part by NSF grant DMS-1600592 and grants 18-01-00353A and 19-01-00682 of the Russian Foundation for Basic Research.

${ }^{\dagger}$ Department of Mathematics, University of Illinois at Urbana-Champaign, IL, USA, mlavrov@illinois.edu.

${ }^{\ddagger}$ University of Califonia, San Diego, La Jolla, CA 92093, USA and University of Illinois at Urbana-Champaign, Urbana, IL 61801, USA. E-mail: ruluo@ucsd.edu. Research is supported in part by NSF grants DMS-1600592 and DMS-1902808.

${ }^{\S}$ University of Illinois at Urbana-Champaign, Urbana, IL 61801, USA. E-mail: zirlin2@illinois.edu. Research is supported in part by Arnold O. Beckman Research Award (UIUC) RB20003.
} 


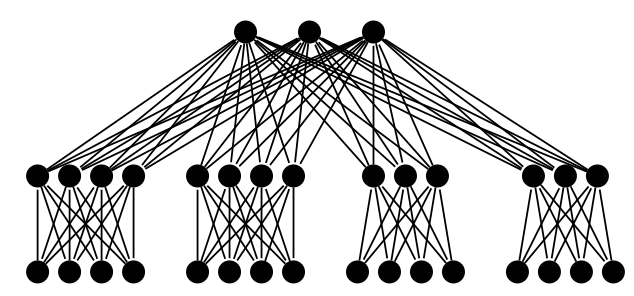

Figure 1: An example of Construction 1.3 .

Construction 1.3. Let $n_{1} \geq n_{2} \geq n_{3} \geq n_{4} \geq 1$ be such that $n_{1}+n_{2}+n_{3}+n_{4}=n$. Let $G_{4}\left(n_{1}, \ldots, n_{4} ; \delta\right) \in \mathcal{G}(n, 4 \delta-9, \delta)$ be the bipartite graph obtained from $\bigcup_{j=1}^{4} K_{\delta-3, n_{j}}$ by adding 3 vertices $a_{1}, a_{2}, a_{3}$, all of which are adjacent to every vertex in the parts of size $n_{1}, n_{2}, n_{3}$, and $n_{4}$. Then a longest cycle in $G_{4}\left(n_{1}, \ldots, n_{4} ; \delta\right)$ has length $2\left(n_{1}+n_{2}+n_{3}\right) \leq 2(n-1)$.

The main result of the paper is that Construction 1.3 is indeed extremal for 3-connected graphs:

Theorem 1.4. Let $m, n, \delta$ be integers. If $\delta \geq \max \left\{n, \frac{m+10}{4}\right\}$, then every 3 -connected graph $G \in$ $\mathcal{G}(n, m, \delta)$ contains a cycle of length $2 n$.

We discuss possible extensions of Theorem 1.4 to $k$-connected bipartite graphs and hypergraphs in concluding remarks. We will apply this theorem in a forthcoming paper on so-called super-pancyclic bipartite graphs and hypergraphs. This notion was introduced and discussed in [7].

In the next section, we discuss how Theorem 1.4 can be translated into the language of hamiltonian Berge cycles.

\subsection{Hamiltonian Berge cycles in hypergraphs}

A hypergraph $\mathcal{H}$ is a set of vertices $V(\mathcal{H})$ and a set of edges $E(\mathcal{H})$ such that each edge is a subset of $V(\mathcal{H})$.

We consider hypergraphs with edges of any size. The degree, $d(v)$, of a vertex $v$ is the number of edges that contain $v$. The minimum degree of a hypergraph $\mathcal{H}$ is $\delta(\mathcal{H}):=\min _{v \in V(\mathcal{H})} d(v)$. The co-degree of a vertex set $A$ is the number of edges that contain $A$.

A Berge cycle of length $\ell$ in a hypergraph is a set of $\ell$ distinct vertices $\left\{v_{1}, \ldots, v_{\ell}\right\}$ and $\ell$ distinct edges $\left\{e_{1}, \ldots, e_{\ell}\right\}$ such that $v_{i}, v_{i+1} \in e_{i}$ for every $i \in[\ell]$ (indices are taken modulo $\ell$ ). The vertices $\left\{v_{1}, \ldots, v_{\ell}\right\}$ are the base vertices of the cycle.

Naturally, a Berge hamiltonian cycle in a hypergraph $\mathcal{H}$ is a Berge cycle whose set of base vertices is $V(\mathcal{H})$.

Let $\mathcal{H}=(V(\mathcal{H}), E(\mathcal{H}))$ be a hypergraph. The incidence graph of $\mathcal{H}$ is the bipartite graph $I(\mathcal{H})$ with parts $(X, Y)$ where $X=V(\mathcal{H}), Y=E(\mathcal{H})$ such that for $e \in Y, v \in X$, ev $\in E(I(\mathcal{H}))$ if and only if the vertex $v$ is contained in the edge $e$ in $\mathcal{H}$.

If $\mathcal{H}$ has $n$ vertices, $m$ edges and minimum degree at least $\delta$, then $I(\mathcal{H}) \in \mathcal{G}(n, m, \delta)$. There is a simple relation between the cycle lengths in a hypergraph $\mathcal{H}$ and its incidence graph $I(\mathcal{H})$ : If $\left\{v_{1}, \ldots, v_{\ell}\right\}$ and $\left\{e_{1}, \ldots, e_{\ell}\right\}$ form a Berge cycle of length $\ell$ in $\mathcal{H}$, then $v_{1} e_{1} \ldots v_{\ell} e_{\ell} v_{1}$ is a cycle of length $2 \ell$ in $I(\mathcal{H})$, and vice versa. 
For a positive integer $k$, call a hypergraph $k$-connected if its incidence graph is $k$-connected.

If one would like to prove an analog of Dirac's theorem on hamiltonian cycles in graphs for hamiltonian Berge cycles in hypergraphs, then the bound on the minimum degree would be exponential in $n$. One of the examples is the following construction from [7].

Construction $1.5([7])$. Let $V(\mathcal{H})=V_{1} \cup V_{2}$ where $\left|V_{1}\right|=\lceil(n+2) / 2\rceil,\left|V_{2}\right|=\lfloor(n-2) / 2\rfloor$, $V_{1} \cap V_{2}=\emptyset$, and let $E(\mathcal{H})=E_{1} \cup E_{2}$, where $E_{1}$ is the set of all subsets $A$ of $V(\mathcal{H})$ of size $\lceil n / 4\rceil$ such that $\left|V_{1} \cap A\right|=1$ (and $\left|V_{2} \cap A\right|=\lceil n / 4\rceil-1$ ), and $E_{2}=\left\{V_{1}\right\}$. Then $\mathcal{H}$ has an exponential in $n$ minimum degree, high connectivity and positive codegree of each pair of the vertices. But $\mathcal{H}$ has no Berge hamiltonian cycle.

On the other hand, rephrasing Theorem 1.4 in terms of hypergraphs, we get a reasonable and sharp bound on the minimum degree in terms of the number of vertices and edges that provides the existence of hamiltonian Berge cycles in 3-connected hypergraphs.

Theorem 1.6. Let positive integers $n, m, \delta$ be such that

$$
\delta \geq \max \left\{n, \frac{m+10}{4}\right\} .
$$

Then every 3-connected $n$-vertex hypergraph with $m$ edges and minimum degree at least $\delta$ has a hamiltonian Berge cycle.

\subsection{Notation and outline of the proof of Theorem 1.4}

For a graph $G$, a cycle $C$ in $G$, and a vertex $x$ not appearing in $C$, let $t(x, C)$ denote the size of a largest $x, V(C)$-fan in $G$, i.e. the largest number of $x, V(C)$-paths such that any two of them share only $x$. Since $G$ is 3 -connected, $t(x, C) \geq 3$.

Our proof is by contradiction. We assume that for some positive integers $m, n, \delta$ with $\delta \geq$ $\max \left\{n, \frac{m+10}{4}\right\}$, there is a counter-example: a 3 -connected $(X, Y)$-bigraph $G \in \mathcal{G}(n, m, \delta)$ with no $2 n$-cycles. We study the properties of $G$.

We consider each cycle $C$ in $G$ equipped with a clockwise direction. For every vertex $u$ of $C$, $x_{C}^{+}(u)$ denotes the closest to $u$ clockwise vertex of $X$ distinct from $u$. For every vertex $u$ of $C$, $x_{C}^{-}(u)$ denotes the closest to $u$ counterclockwise vertex of $X$ distinct from $u$. For a set $U \subset V(C)$, $X_{C}^{+}(U)=\left\{x_{C}^{+}(u): u \in U\right\}$. When $C$ is clear from the context, the subscripts could be omitted. The vertices $y^{+}(u), y^{-}(u)$ and the sets $X^{-}(U), Y^{+}(U), Y^{-}(U)$ are defined similarly.

We consider triples $(C, x, F)$ where $C$ is a cycle, $x \in X-V(C)$ and $F$ is an $x, C$-fan. By $D(C, x)$ we will denote the component of $G-C$ containing $x$. By definition, $V(F)-V(C) \subseteq D(C, x)$.

Definition 1.7. A triple $(C, x, F)$ is better than a triple $\left(C^{\prime}, x^{\prime}, F^{\prime}\right)$ if

(a) $|C|>\left|C^{\prime}\right|$, or

(b) $|C|=\left|C^{\prime}\right|$ and $t(x, C)>t\left(x^{\prime}, C^{\prime}\right)$, or

(c) $|C|=\left|C^{\prime}\right|, t(x, C)=t\left(x^{\prime}, C^{\prime}\right)$, and $|V(F) \cap V(C) \cap Y|>\left|V\left(F^{\prime}\right) \cap V\left(C^{\prime}\right) \cap Y\right|$, or

(d) $|C|=\left|C^{\prime}\right|, t(x, C)=t\left(x^{\prime}, C^{\prime}\right),|V(F) \cap V(C) \cap Y|=\left|V\left(F^{\prime}\right) \cap V\left(C^{\prime}\right) \cap Y\right|$, and $|V(F)|<\left|V\left(F^{\prime}\right)\right|$, or 
(e) $|C|=\left|C^{\prime}\right|, t(x, C)=t\left(x^{\prime}, C^{\prime}\right),|V(F) \cap V(C) \cap Y|>\left|V\left(F^{\prime}\right) \cap V\left(C^{\prime}\right) \cap Y\right|,|V(F)|=\left|V\left(F^{\prime}\right)\right|$ and $|V(D(C, x))|<\left|V\left(D\left(C^{\prime}, x\right)\right)\right|$.

Choose a best triple $(C, x, F)$. Let

$$
\begin{aligned}
2 \ell=|C|, & t=t(x, C), & T=T(C, x, F)=V(F) \cap V(C), \\
t_{X} & =|T \cap X|, & t_{Y}=|T \cap Y| .
\end{aligned}
$$

Similarly, let $\widetilde{T}=\widetilde{T}(C, x)$ be the set of all vertices of $C$ adjacent to a vertex of $D(C, x)$, and let $\widetilde{t}=\widetilde{t}(C, x)=|\widetilde{T}|$. By definition, $\widetilde{T} \supseteq T$ and $\widetilde{t} \geq t$. Viewing $F$ as a tree (spider) with root $x$, any two vertices $u, v \in V(F)$ define the unique $u, v$-path $F[u, v]$ in $F$. For $u, v \in V(C)$, let $C[u, v]$ be the clockwise $u, v$-path in $C$ and let $C^{-}[u, v]$ be the counterclockwise $u, v$-path in $C$. If $D=D(C, x)$ and $u, v \in D \cup \widetilde{T}(C, x)$, then let $P_{D}[u, v]$ be a longest $u, v$-path all of whose internal vertices are in $D$.

We will analyze the properties of best triples $(C, x, F)$ and in all cases will come to a contradiction, either by finding a better triple or by proving that $m \geq 4 \delta-9$. For this, we will try to construct so called good subsets $W$ of $X \cap T$, defined later, such that total neighborhood of $W \cup\{x\}$ will be too large. One feature of a good set will be that no two members of such set have a common neighbor outside of $C, \mathbf{C O N}$ for short.

In the next section we prove basic properties of our best triple $(C, x, F)$. Then in Section 3 we show that $t=\widetilde{t}=3$. Since $G$ is 3 -connected, this means that for every $x^{\prime} \in X-C, t\left(x^{\prime}, C\right)=3$. In Section 3.1, we discuss special types of components of $G-C$ and possibilities to choose a triple $(C, x, F)$ with $x$ in such a component. After that we consider $T=T(C, x, F)$ and try to find a 4-element good subset of the set $A=X^{+}(T) \cup X^{-}(T)$. The main obstacles will be that some members of $A$ have many common neighbors, in particular, CONs. Section 4 is devoted to the case analysis of different types of such CONs. We conclude the paper with some comments.

\section{Preliminary lemmas}

Lemma 2.1. The following inequalities always hold:

$$
\text { (i) } \ell \geq t+t_{X} ; \quad \text { (ii) }|X|-\ell+t_{X} \geq 3 ; \quad \text { (iii) }|X| \geq t+3 .
$$

Proof. If $w \in T \cap X$ and $y^{+}(w) \in T$, then the cycle $w F\left[w, y^{+}(w)\right] y^{+}(w) C\left[y^{+}(w), w\right] w$ is longer than $C$, a contradiction. Similarly, $y^{-}(w), x^{+}(w), x^{-}(w) \notin T$. Thus, $t_{X} \leq \ell / 2$ and $t_{Y} \leq \ell-2 t_{X}$. This proves (i).

Since $\delta \geq|X| \geq \ell+1 \geq t+1=d_{F}(x)+1$, there is $y \in N(x)-N_{F}(x)$. By (d) in the definition of $(C, x, F), y \notin V(F)$. By the maximality of $t, y \notin V(C)-V(F)$. Since $G$ is 3-connected, $G-x$ has a $y, C$-fan $F^{\prime}$ of size 2 . Let $x^{\prime}, x^{\prime \prime}$ be the neighbors of $y$ in $F^{\prime}$. If, say $x^{\prime} \in V(C)$, then by the maximality of $t, x^{\prime} \in T$. Thus $\left\{x, x^{\prime}, x^{\prime \prime}\right\} \subset(X-V(C)) \cup(T \cap X)$. This yields (ii). Now (i) and (ii) together imply (iii).

Lemma 2.2. If $w \in \widetilde{T} \cap X$, then

(i) $y^{+}(w) \notin \widetilde{T}$ and 
(ii) $y^{+}(w)$ has no neighbors in $X^{+}(\widetilde{T})-x^{+}(w)$.

Proof. If $y^{+}(w)$ has a neighbor in $D=D(C, x)$, then the cycle $w P_{D}\left[w, y^{+}(w)\right] y^{+}(w) C\left[y^{+}(w), w\right] w$ is longer than $C$. This contradiction proves (i).

Suppose $y^{+}(w) u \in E(G)$ for some $u \in X^{+}(\widetilde{T})-x^{+}(w)$. Let $u=x^{+}(v)$ for $v \in \widetilde{T}-w$. Consider the cycle $C^{\prime}=w C^{-}[w, u] u y^{+}(w) C\left[y^{+}(w), v\right] v P_{D}[v, w] w$. Then $C^{\prime}$ is longer than $C$, unless $v \in X$ and $v$ and $w$ have a common neighbor $y$ in $D$. In the last case, $\left|C^{\prime}\right|=|C|$ and the only vertex in $V(C)-V\left(C^{\prime}\right)$ is $y^{+}(v)$ which by (i) does not have neighbors in $D$. Define an $x, C^{\prime}$-fan $F^{\prime}$ as follows. If $y \notin V(F)$, then let $F^{\prime}=F$. If $y \in V(F)$, say $y \in F\left[x, u_{i}\right]$ for some $u_{i} \in T$, then let $F^{\prime}=F-E\left(F\left[y, u_{i}\right]\right)$. In both cases, since $y^{+}(v)$ does not have neighbors in $D\left(C^{\prime}, x\right) \subset D$, the triple $\left(C^{\prime}, x, F^{\prime}\right)$ is better than $(C, x, F)$ : if $y \notin V(F)$, then by (e), otherwise either by (c) or by (d).

Lemma 2.3. If $x_{1} \in X^{+}(\widetilde{T})$, then $x_{1}$ cannot have a neighbor in $D=D(C, x)$, i.e., $x_{1} \notin \widetilde{T}$.

Proof. Suppose $x_{1}$ has a neighbor $y^{\prime}$ in $D$. Let $u_{1} \in \widetilde{T}$ be such that $x_{1}=x^{+}\left(u_{1}\right)$ and $z$ be a neighbor of $u_{1}$ in $D$. Let $P$ be a $z, y^{\prime}$-path in $D$ and the cycle $C^{\prime}$ be defined by $C^{\prime}=x_{1} C\left[x_{1}, u_{1}\right] u_{1} z P y^{\prime} x_{1}$. If $y^{\prime} \neq z$, then $C^{\prime}$ is longer than $C$ and we are done. Thus $z=y^{\prime}$ and hence $u_{1} \in X$. In this case $C^{\prime}$ and $C$ have the same length and $t\left(x, C^{\prime}\right)=t(x, C)$. As in the proof of Lemma 2.2(ii), if $y^{\prime} \notin V(F)$, then let $F^{\prime}=F$. If $y^{\prime} \in V(F)$, say $y^{\prime} \in F\left[x, u_{i}\right]$ for some $u_{i} \in T$, then let $F^{\prime}=F-E\left(F\left[y^{\prime}, u_{i}\right]\right)$. In both cases, since by Lemma 2.2(i), $y^{+}\left(u_{1}\right)$ does not have neighbors in $D\left(C^{\prime}, x\right) \subset D$, the triple $\left(C^{\prime}, x, F^{\prime}\right)$ is better than $(C, x, F)$ : if $y^{\prime} \notin V(F)$, then by (e), otherwise either by (c) or by (d).

Given a cycle $C$ and distinct $x_{1}, x_{2}, x_{3} \in X \cap V(C)$, we say that $x_{1}$ and $x_{2}$ cross at $x_{3}$ if the cyclic order is $x_{1}, x_{3}, x_{2}$ and $x_{1} y^{+}\left(x_{3}\right), x_{2} y^{-}\left(x_{3}\right) \in E(G)$ or if the cyclic order is $x_{1}, x_{2}, x_{3}$ and $x_{1} y^{-}\left(x_{3}\right), x_{2} y^{+}\left(x_{3}\right) \in E(G)$. In this case, we also say that $x_{3}$ is crossed by $x_{1}$ and $x_{2}$.

Lemma 2.4. Suppose that $x_{1}, x_{2} \in X^{+}(\widetilde{T})$, cross at $x_{3} \in X \cap V(C)$. Then $x_{3} \notin \widetilde{T}$.

Proof. Suppose that the cyclic order is $x_{1}, x_{3}, x_{2}$ and $x_{1} y^{+}\left(x_{3}\right), x_{2} y^{-}\left(x_{3}\right) \in E(G)$ (the other case is symmetric). Let $y$ be a neighbor of $x_{3}$ in $D$. Let $u_{1} \in \widetilde{T}$ be such that $x_{1}=x^{+}\left(u_{1}\right)$ and $z$ be a neighbor of $u_{1}$ in $D$. Let $P$ be a $z, y$-path in $D$ and the cycle $C^{\prime}$ be defined by

$$
C^{\prime}:=x_{1} y^{+}\left(x_{3}\right) C\left[y^{+}\left(x_{3}\right), u_{1}\right] u_{1} z P y x_{3} C^{-}\left[x_{3}, x_{1}\right] x_{1} .
$$

If $y \neq z$, then $C^{\prime}$ is longer than $C$ and we are done. Thus $z=y$. In this case, $C^{\prime}$ and $C$ have the same length and $t\left(x, C^{\prime}\right)=t(x, C)$. As in the proof of Lemma 2.2(ii), if $y \notin V(F)$, then let $F^{\prime}=F$. If $y \in V(F)$, say $y \in F\left[x, u_{i}\right]$ for some $u_{i} \in T$, then let $F^{\prime}=F-E\left(F\left[y, u_{i}\right]\right)$. Again as in the proof of Lemma 2.2, the triple $\left(C^{\prime}, x, F^{\prime}\right)$ is better than $(C, x, F)$.

Recall that for two vertices in $G$, CON means "a common neighbor outside of $C . "$

Lemma 2.5. Suppose that $x_{1}, x_{2} \in X^{+}(\widetilde{T})$. Then

(i) $x_{1}$ and $x_{2}$ have no CON;

(ii) neither of $x_{1}$ and $x_{2}$ has a CON with $x$. 
Proof. Part (ii) follows from Lemma 2.3. So, suppose $x_{1}$ and $x_{2}$ have a CON $y$, and $u_{1}, u_{2} \in \widetilde{T}$ are such that $x_{1}=x^{+}\left(u_{1}\right)$ and $x_{2}=x^{+}\left(u_{2}\right)$. By Lemma 2.3, $y \notin D$. Consider the cycle

$$
C^{\prime}:=x_{1} C\left[x_{1}, u_{2}\right] u_{2} P_{D}\left[u_{2}, u_{1}\right] u_{1} C^{-}\left[u_{1}, x_{2}\right] x_{2} y x_{1} .
$$

Cycle $C^{\prime}$ is longer than $C$, unless $u_{1}, u_{2} \in X$ and have a common neighbor $y^{\prime}$ in $D$. In the last case, $\left|C^{\prime}\right|=|C|$ and the only vertices in $V(C)-V\left(C^{\prime}\right)$ are $y^{+}\left(u_{1}\right)$ and $y^{+}\left(u_{1}\right)$ which by Lemma 2.3(i) do not have neighbors in $D$. Define an $x, C^{\prime}$-fan $F^{\prime}$ as follows. If $y^{\prime} \notin V(F)$, then let $F^{\prime}=F$. If $y^{\prime} \in V(F)$, say $y^{\prime} \in F\left[x, u_{i}\right]$ for some $u_{i} \in T$, then let $F^{\prime}=F-E\left(F\left[y^{\prime}, u_{i}\right]\right)$. In both cases, since $y^{+}\left(u_{1}\right)$ and $y^{+}\left(u_{2}\right)$ do not have neighbors in $D\left(C^{\prime}, x\right) \subset D$, the triple $\left(C^{\prime}, x, F^{\prime}\right)$ is better than $(C, x, F)$ : if $y \notin V(F)$, then by (e), otherwise either by (c) or by (d).

Lemma 2.6. Suppose $u_{1}, u_{2} \in \widetilde{T}$ are such that the path $P_{D}\left[u_{1}, u_{2}\right]$ contains an internal vertex in $X$. If $x_{1}=x^{+}\left(u_{1}\right)$ and $x_{2}=x^{+}\left(u_{2}\right)$ cross at $x_{3} \in X \cap V(C)$, then

(i) $x_{3} \notin \widetilde{T}$ and if $x_{3}=x^{+}(u)$ where $u \in \widetilde{T}$, then $u \in Y$;

(ii) $G$ has a cycle $C^{\prime}$ containing $\left(X \cap V(C)-x_{3}\right) \cup\left(X \cap P_{D}\left[u_{1}, u_{2}\right]\right)$ such that $\left|C^{\prime}\right| \geq|C|$;

(iii) $x_{3}$ has no CON with any vertex in the set $\{x\} \cup X^{+}(T)$;

(iv) $x_{3}$ has at most $t$ neighbors on $C$.

Proof. Part (i) follows from Lemmas 2.2 and 2.4. The cycle

$$
C_{1}:=x_{1} y^{+}\left(x_{3}\right) C\left[y^{+}\left(x_{3}\right), u_{2}\right] u_{2} P_{D}\left[u_{2}, u_{1}\right] u_{1} C^{-}\left[u_{1}, x_{2}\right] x_{2} y^{-}\left(x_{3}\right) C^{-}\left[y^{-}\left(x_{3}\right), x_{1}\right] x_{1}
$$

proves (ii).

To prove (iii), assume that $y$ is a CON of $x_{3}$ with a vertex in $\{x\} \cup X^{+}(T)$, and consider all cases. First note that by Lemma 2.4. $y \notin D$; in particular, $x_{3}$ has no CON with $x$. If $u_{j} \in \widetilde{T}, x_{j}=x^{+}\left(u_{j}\right)$, $y x_{j} \in E(G)$, and $x_{j} \in C\left[y^{+}\left(x_{3}\right), u_{1}\right]$, then the cycle

$$
C^{\prime}:=x_{1} C\left[x_{1}, x_{3}\right] x_{3} y x_{j} C\left[x_{j}, u_{1}\right] u_{1} P_{D}\left[u_{1}, u_{j}\right] u_{j} C^{-}\left[u_{j}, y^{+}\left(x_{3}\right)\right] y^{+}\left(x_{3}\right) x_{1}
$$

is longer than $C$, unless $P_{D}\left[u_{1}, u_{j}\right]=u_{1} y^{\prime} u_{j}$ for some $y^{\prime} \in D$. If $P_{D}\left[u_{1}, u_{j}\right]=u_{1} y^{\prime} u_{j}$, then $\left|C^{\prime}\right|=|C|$ and the only vertices in $V(C)-V\left(C^{\prime}\right)$ are $y^{+}\left(u_{1}\right)$ and $y^{+}\left(u_{j}\right)$ which by Lemma 2.3(i) do not have neighbors in $D$. Define an $x, C^{\prime}$-fan $F^{\prime}$ as at the end of the proof of Lemma 2.3 , and see that $\left(C^{\prime}, x, F^{\prime}\right)$ is better than $(C, x, F)$ exactly as there. Similarly, if $x_{j} \in C\left[u_{1}, y^{-}\left(x_{3}\right)\right]$, then the cycle

$$
C^{\prime}:=x_{2} C\left[x_{2}, u_{j}\right] u_{j} P_{D}\left[u_{j}, u_{2}\right] u_{2} C^{-}\left[u_{2}, x_{3}\right] x_{3} y x_{j} C\left[x_{j}, y^{-}\left(x_{3}\right)\right] y^{-}\left(x_{3}\right) x_{2}
$$

is longer than $C$, unless $P_{D}\left[u_{j}, u_{2}\right]=u_{j} y^{\prime} u_{2}$ for some $y^{\prime} \in D$. Again, defining $F^{\prime}$ as above, we get a triple $\left(C^{\prime}, x, F^{\prime}\right)$ better than $(C, x, F)$, a contradiction. This proves (iii).

By the choice of $(C, x, F)$ and (ii), $x_{3}$ has at most $t$ neighbors on $C_{1}$. The only vertices in $Y \cap$ $V(C)-V\left(C_{1}\right)$ are $y^{-}\left(x_{1}\right)$ and $y^{-}\left(x_{2}\right)$. If $x_{3} y^{-}\left(x_{1}\right) \in E(G)$, then the cycle

$$
y^{-}\left(x_{1}\right) C\left[y^{-}\left(x_{1}\right), y^{-}\left(x_{3}\right)\right] y^{-}\left(x_{3}\right) x_{2} C\left[x_{2}, u_{1}\right] u_{1} P_{D}\left[u_{1}, u_{2}\right] u_{2} C^{-}\left[u_{2}, x_{3}\right] x_{3} y^{-}\left(x_{1}\right)
$$



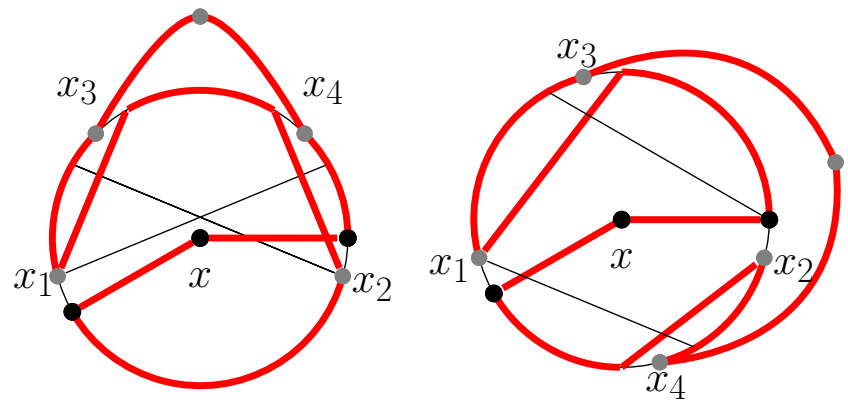

Figure 2: Longer cycles when $x_{1}$ and $x_{2}$ in Lemma 2.7 have multiple crossings.

is longer than $C$. If $x_{3} y^{-}\left(x_{2}\right) \in E(G)$, then the cycle

$$
x_{1} C\left[x_{1}, x_{3}\right] x_{3} y^{-}\left(x_{2}\right) C\left[y^{-}\left(x_{2}\right), u_{1}\right] u_{1} P_{D}\left[u_{1}, u_{2}\right] u_{2} C^{-}\left[u_{2}, y^{+}\left(x_{3}\right)\right] y^{+}\left(x_{3}\right) x_{1}
$$

is longer than $C$. This proves (iv).

Lemma 2.7. Suppose $u_{1}, u_{2} \in \widetilde{T}$ are such that the path $P_{D}\left[u_{1}, u_{2}\right]$ contains an internal vertex in $X, x_{1}=x^{+}\left(u_{1}\right)$, and $x_{2}=x^{+}\left(u_{2}\right)$. Then at most one vertex in $C$ is crossed by $x_{1}$ and $x_{2}$.

Proof. Suppose vertices $x_{3}, x_{4} \in V(C) \cap X$ are crossed by $x_{1}$ and $x_{2}$. We will show first that $x_{3}$ and $x_{4}$ have no CON. Suppose there is some $y \in\left(N\left(x_{3}\right) \cap N\left(x_{4}\right)\right)-V(C)$. By Lemma 2.6, $y \notin V(D)$.

We consider two cases. If $x_{3}$ and $x_{4}$ both are on $C\left[x_{1}, x_{2}\right]$ or both are on $C\left[x_{2}, x_{1}\right]$, then we may assume that their cyclic order is $x_{1}, x_{3}, x_{4}, x_{2}$. In this case, the cycle

$$
x_{1} C\left[x_{1}, x_{3}\right] x_{3} y x_{4} C\left[x_{4}, u_{2}\right] u_{2} P_{D}\left[u_{2}, u_{1}\right] u_{1} C^{-}\left[u_{1}, x_{2}\right] x_{2} y^{-}\left(x_{4}\right) C^{-}\left[y^{-}\left(x_{4}\right), y^{+}\left(x_{3}\right)\right] y^{+}\left(x_{3}\right) x_{1}
$$

(see Figure 2, left) is longer than $C$.

If one of $x_{3}$ and $x_{4}$ is on $C\left[x_{1}, x_{2}\right]$ and the other is on $C\left[x_{2}, x_{1}\right]$, then we may assume that their cyclic order is $x_{1}, x_{3}, x_{2}, x_{4}$. In this case, the cycle

$$
x_{1} C\left[x_{1}, x_{3}\right] x_{3} y x_{4} C^{-}\left[x_{4}, x_{2}\right] x_{2} y^{+}\left(x_{4}\right) C\left[y^{+}\left(x_{4}\right), u_{1}\right] u_{1} P_{D}\left[u_{1}, u_{2}\right] u_{2} C^{-}\left[u_{2}, y^{+}\left(x_{3}\right)\right] y^{+}\left(x_{3}\right) x_{1}
$$

(see Figure 2 right) is longer than $C$. This proves that $x_{3}$ and $x_{4}$ have no CON.

Let $A=X^{+}(T) \cup\left\{x, x_{3}, x_{4}\right\}$ (possibly, $X^{+}(T) \cap\left\{x_{3}, x_{4}\right\} \neq \emptyset$ ), and $A^{\prime}=A-\left\{x, x_{3}, x_{4}\right\}$. Note that $\left|A^{\prime}\right| \geq t-2$.

By definition, $|N(x)-C| \geq \delta-t_{Y}$. By Lemma 2.6(iv), $\left|N\left(x_{3}\right)-C\right| \geq \delta-t$ and $\left|N\left(x_{4}\right)-C\right| \geq \delta-t$. By Lemma 2.2,

$$
\sum_{u \in A^{\prime}}|N(u) \cap V(C)| \leq \ell\left|A^{\prime}\right|-t_{X}\left|A^{\prime}\right|+\min \left\{t_{X},\left|A^{\prime}\right|\right\} .
$$

By Lemmas 2.5 and 2.6(iii), no two distinct vertices in $A$ have a CON. Thus, using (2) and remembering about the $\ell$ vertices in $Y \cap V(C)$, we get

$$
|Y| \geq \ell+\sum_{u \in A}|N(u)-V(C)|
$$




$$
\begin{aligned}
& =\ell+|N(x)-V(C)|+\left|N\left(x_{3}\right)-V(C)\right|+\left|N\left(x_{4}\right)-V(C)\right|+\sum_{u \in A^{\prime}}|N(u)-V(C)| \\
& \geq \ell+\left(\delta-t_{Y}\right)+(\delta-t)+(\delta-t)+\left(\delta\left|A^{\prime}\right|-\sum_{u \in A^{\prime}}|N(u) \cap V(C)|\right) \\
& \geq \ell+\left(\left|A^{\prime}\right|+3\right) \delta-2 t-t_{Y}-\left(\ell-t_{X}\right)\left|A^{\prime}\right|-\min \left\{t_{X},\left|A^{\prime}\right|\right\} \\
& \geq \ell+(t-2+3) \delta-2 t-\left(t-t_{X}\right)-\left(\ell-t_{X}\right)(t-2)-\min \left\{t_{X}, t-2\right\} \\
& =\ell+(t+1) \delta-\left(3 t-t_{X}\right)-\left(\ell-t_{X}\right)(t-2)-\min \left\{t_{X}, t-2\right\} \\
& \geq \ell+(t+1) \delta-3 t-\left(\ell-t_{X}\right)(t-3+1) \\
& =(t+1) \delta-3 t-\left(\ell-t_{X}\right)(t-3)+t_{X} .
\end{aligned}
$$

Since by Lemma 2.1, $\delta \geq \ell-t_{X}+3$, this yields

$$
|Y| \geq(t+1) \delta-3 t-\delta(t-3)+3(t-3)+t_{X}=4 \delta-9+t_{X}
$$

This contradiction proves the lemma.

The following lemma holds for any bipartite graph $G$ (no restrictions on minimum degree or connectivity).

Lemma 2.8. Let $C$ be a cycle of $G$, and let $u, v \in V(C) \cap X$. If $u$ and $v$ have at most a crossings, then $d_{C}(u)+d_{C}(v) \leq|V(C)| / 2+2+a$.

Proof. We induct on $a$. Suppose $a=0$. Consider the two paths $P_{1}=C[u, v]$ and $P_{2}=C^{-}[u, v]$. In $P_{1}=v_{1} \ldots v_{k}\left(v_{1}=u, v_{k}=v\right)$, each $v_{i} \in X$ satisfies at most one of the following: $v_{i+1} u \in E(G)$ or $v_{i-1} v \in E(G)$. So $d_{P_{1}}(u)+d_{P_{1}}(v) \leq\left|V\left(P_{1}\right) \cap X\right|$. Similarly, $d_{P_{2}}(u)+d_{P_{2}}(v) \leq\left|V\left(P_{2}\right) \cap X\right|$. Since $\left(X \cap V\left(P_{1}\right)\right) \cap\left(X \cap V\left(P_{2}\right)\right)=\{u, v\}$ and $V\left(P_{1}\right) \cup V\left(P_{2}\right)=V(C)$, we get $d_{C}(u)+d_{C}(v) \leq|V(C)| / 2+2$.

For $a \geq 1$, delete an edge incident to $u$ that is used in a crossing, and apply induction.

\section{Bounds on $t$ and $\widetilde{t}$ in best triples}

Recall that $(C, x, F)$ is a best triple, $D=D(C, x)$ is the component of $G-V(C)$ containing $x$, $T=V(F) \cap V(C)$, and $\widetilde{T}=N_{C}(D)$.

A set of vertices $W=\left\{x_{1}, \ldots, x_{k}\right\} \subseteq X \cap V(C)$ is good if

(i) $d_{C}(x) \leq k$,

(ii) the vertices of $\{x\} \cup W$ pairwise have no CON, and

(iii) we can partition $W$ into sets $W_{1}, \ldots, W_{s}$ such that for each $j \in[s],\left|W_{j}\right| \geq 2$ and any two distinct vertices in $W_{j}$ cross at no more than one vertex in $C$.

Lemma 3.1. If $W$ is a good set, then $|W|<\max \{4, t\}$.

Proof. Suppose $k \geq \max \{4, t\}$ and $W=\left\{x_{1}, \ldots, x_{k}\right\}$ is a good set. Note that $\delta \geq|X| \geq|W| \geq 4$. Let $\left(W_{1}, \ldots, W_{s}\right)$ be a partition of $W$ satisfying (iii) in the definition of a good set. By Lemma 2.8, 
if $x_{i}$ and $x_{j}$ have at most one crossing, then $d_{C}\left(x_{i}\right)+d_{C}\left(x_{j}\right) \leq \ell+3$. Hence

$$
\sum_{i=1}^{k} d_{C}\left(x_{i}\right)=\sum_{j=1}^{s} \sum_{w \in W_{j}} d_{C}(w) \leq k(\ell+3) / 2 .
$$

Since $|Y \cap V(C)|=\ell, \delta(G) \geq \delta$ and $k \geq t$, we get

$$
|Y| \geq \ell+(k+1) \delta-t-k \frac{\ell+3}{2} \geq \ell\left(1-\frac{k}{2}\right)+k\left(\delta-\frac{5}{2}\right)+\delta .
$$

Since the net coefficient at $\ell$ is negative and $\ell \leq|X|-1 \leq \delta-1$, this is at least $k\left(\frac{\delta}{2}-2\right)+2 \delta-1$. Now the net coefficient at $k$ is nonnegative, so the minimum is attained at $k=4$. Hence $|Y| \geq 4 \delta-9$, a contradiction.

Next, we show that both $t$ and $\widetilde{t}$ are small.

Lemma 3.2. $t=3$.

Proof. Since $G$ is 3 -connected, $t=|T| \geq 3$. Suppose $t \geq 4$. We claim that $X^{+}(T)$ is a good set.

Since $F$ is a largest $x, C$-fan, $x$ has at most $t$ neighbors in $C$. By Lemma 2.7, for any $x_{i}, x_{j} \in X^{+}(T)$, $x_{i}$ and $x_{j}$ have at most one crossing in $C$. By Lemma 2.5, no two distinct vertices in $X^{+}(T) \cup\{x\}$ have a CON. This certifies that $X^{+}(T)$ is good, a contradiction to Lemma 3.1 .

Lemma 3.3. $|\widetilde{T}|=3$.

Proof. We have $T \subseteq \widetilde{T}$. Suppose $|\widetilde{T}| \geq 4$. Choose a set $U=\left\{u_{1}, \ldots, u_{4}\right\} \subseteq \widetilde{T}$ so that $T=$ $\left\{u_{1}, u_{2}, u_{3}\right\}$, and $u_{4} \in \widetilde{T}-T$. Let $P$ be a shortest path from $u_{4}$ to $F-C$ in $G\left[D+u_{4}\right]$. Let $j \in[3]$ be such that the end, $p$, of $P$ distinct from $u_{4}$ belongs to the $x, u_{j}$-path in $F$. Assume $[3]=\left\{j, j^{\prime}, j^{\prime \prime}\right\}$. The path $u_{j^{\prime}} F\left[u_{j^{\prime}}, p\right] p P u_{4}$ contains an internal vertex in $X$ (namely, $x$ ). Partition $U$ into $U^{\prime}=\left\{u_{4}, u_{j^{\prime}}\right\}$ and $U^{\prime \prime}=\left\{u_{j}, u_{j^{\prime \prime}}\right\}$.

By Lemma 2.7, each of the pairs $U^{\prime}$ and $U^{\prime \prime}$ has at most one crossing in $C$. Since $F$ is a largest $x, C$-fan, $x$ has at most $t$ neighbors in $C$. By Lemma 2.5, no two distinct vertices in $X^{+}(U) \cup\{x\}$ have a CON. This certifies that $X^{+}(U)$ is good, a contradiction to Lemma 3.1 .

Remark 3.4. Lemma 3.3 implies that $T=\widetilde{T}$, i.e., the only vertices in $C$ with neighbors in $D$ are the vertices of $T$. In particular, no vertex in $V(C)-T$ has a CON with $x$.

\subsection{More structure and fewer crossings}

One of the results of this section is that for any best triple $(C, x, F)$, no vertices in $X^{+}(T)$ cross in $C$. Recall that by Lemma $3.2,|T|=|V(F) \cap V(C)|=3$.

A component $D$ of $V(G)-C$ is 2-rich if there is a set $U=\left\{u_{1}, u_{2}, u_{3}\right\}=V(C) \cap N(D)$ such that for all distinct $i, j, D$ contains a $u_{i}, u_{j}$-path with at least two internal vertices in $X$.

Lemma 3.5. If $|T \cap X| \leq 1$, then $D$ is 2-rich. 
Proof. Suppose $T=\left\{u_{1}, u_{2}, u_{3}\right\}$ where $u_{1}, u_{2} \in Y$. If some $y \in D \cap Y$ is not adjacent to $u_{3}$, then all $y, C$-paths contain internal vertices in $X$, and hence $D$ is 2-rich. Thus we may assume that each $y \in D \cap Y$ is adjacent to $u_{3}$. In particular, $u_{3} \in X$.

By Rule (d) of Definition 1.7, $d_{F}(x)=t=3$, so because $\delta \geq|X|+1 \geq t+3+1 \geq 7$, there is $y^{\prime} \in N(x)$ with $y^{\prime} x \notin E(F)$. Since $G$ is 3 -connected, it contains a $y^{\prime}, C$-fan $F^{\prime}$ with 3 paths. Recall that $y^{\prime} u_{3}$ is one of such paths. For $i=1,2$, let $P_{i}$ be the $y^{\prime}, u_{i}$-path in $F^{\prime}$ and $v_{i} y^{\prime} \in E\left(P_{i}\right)$. Suppose that for $i=1,2$, there is $y_{i} \in N\left(v_{i}\right)-C-y^{\prime}-P_{3-i}$ (possibly, $y_{2}=y_{1}$ ). Then $D$ is 2-rich: $P_{1} \cup P_{2}$ connects $u_{1}$ with $u_{2}$, and for $i \in\{1,2\}$, path $u_{3} y_{i} v_{i} y^{\prime} P_{3-i}$ connects $u_{3}$ with $u_{3-i}$; and each of these three paths contains $\left\{v_{1}, v_{2}\right\} \subset X$. Hence by symmetry we may assume that every neighbor of $v_{1}$ is in $V(C) \cup P_{2}$. Note $N\left(v_{1}\right) \cap V(C) \subseteq\left\{u_{1}, u_{2}, u_{3}\right\}$, since $|\widetilde{T}|=3$. Then the cycle $v_{1} y^{\prime} P_{2} C\left[u_{2}, u_{1}\right] v_{1}$ has at least $2 \delta$ vertices, a contradiction.

Lemma 3.6. Suppose $D$ is not 2-rich. For any $x^{\prime} \in X \cap V(C), G-x^{\prime}$ has no cycle $C^{\prime}$ such that

(i) $X \cap V\left(C^{\prime}\right) \supseteq X \cap V(C)-x^{\prime}+x$, and

(ii) $C^{\prime}$ contains the neighbors $y^{+}\left(x^{\prime}\right)$ and $y^{-}\left(x^{\prime}\right)$ of $x^{\prime}$ on $C$.

Proof. Suppose we have $C^{\prime}$ satisfying (i) and (ii). If we have strict containment in (i), then $\left|C^{\prime}\right|>$ $|C|$, contradicting (a) in the choice of $(C, x, F)$. Thus $X \cap V\left(C^{\prime}\right)=X \cap V(C)-x^{\prime}+x$.

Let $D^{\prime}$ be the component of $G-V\left(C^{\prime}\right)$ containing $x^{\prime}$. Let $M$ be the set of neighbors of $D^{\prime}$ on $C^{\prime}$. By (ii), $\left\{y^{+}\left(x^{\prime}\right), y^{-}\left(x^{\prime}\right)\right\} \subset M$. Since $G$ is 3-connected, $D^{\prime}-\left\{y^{+}\left(x^{\prime}\right), y^{-}\left(x^{\prime}\right)\right\}$ contains an $x^{\prime}, C^{\prime}$-path $P$. Then $P$ together with the edges $x^{\prime} y^{+}\left(x^{\prime}\right)$ and $x^{\prime} y^{-}\left(x^{\prime}\right)$ forms an $x^{\prime}, C^{\prime}$-fan $F^{\prime}$ with $\left|V\left(F^{\prime}\right) \cap V\left(C^{\prime}\right) \cap Y\right| \geq 2$. Moreover since $D$ was not 2-rich, by Lemma 3.5, $|V(F) \cap V(C) \cap Y| \leq 1$. So $\left(C^{\prime}, x^{\prime}, F^{\prime}\right)$ is a better triple than $(C, x, F)$, a contradiction.

Lemma 3.7. No two vertices in $X^{+}(T)$ cross in $C$.

Proof. Suppose $x_{i}=x^{+}\left(u_{i}\right)$ and $x_{j}=x^{+}\left(u_{j}\right)$ cross at some vertex $x_{0} \in V(C) \cap X$. By symmetry, we may assume that their cyclic order is $x_{i}, x_{0}, x_{j}$. Let

$$
C^{\prime}:=x_{i} C\left[x_{i} y^{-}\left(x_{0}\right)\right] y^{-}\left(x_{0}\right) x_{j} C\left[x_{j}, u_{i}\right] u_{i} P_{D}\left[u_{i}, u_{j}\right] u_{j} C^{-}\left[u_{j}, y^{+}\left(x_{0}\right)\right] y^{+}\left(x_{0}\right) x_{i} .
$$

If $D$ is 2-rich, then $P_{D}\left[u_{i}, u_{j}\right]$ has at least 2 internal vertices in $X$, and so $C^{\prime}$ is longer than $C$. If $D$ is not 2-rich, then $C^{\prime}$ satisfies conditions (i) and (ii) of Lemma 3.6, a contradiction.

Let $e$ be an edge of $C$, let $u, v \in V(C)$, and let $P$ be any $u, v$-path containing $e$, which we orient from $u$ to $v$. We say that $P$ and $C$ agree on the edge $e$ if the orientation of $e$ (oriented from $u$ to $v$ ) in the $u, v$-segment of $C$ containing $e$ is the same as the orientation of $e$ in $P$.

Lemma 3.8. Let $u, v \in X \cap V(C)$. Suppose that there is a $u$, v-path $P$ with $(X \cap V(C)) \cup\{x\} \subseteq V(P)$ and there exists some $z, z^{\prime} \in V(P)$ such that $V(P) \cap V(D)=V\left(P\left[z, z^{\prime}\right]\right)$, i.e., $P$ enters and leaves $D$ exactly once. Then

(i) $u$ and $v$ have no common neighbor outside of $P$, and

(ii) if $P$ and $C$ agree on an edge $e$, then $u$ and $v$ cannot have a crossing at an endpoint of $e$. 
Proof. Note that $x \in P\left[z, z^{\prime}\right]$. If $u$ and $v$ had a common neighbor outside $P$, then we could extend $P$ to a cycle longer than $C$, so (i) holds.

To prove (ii), suppose that $P$ and $C$ agree on an edge $e$ which lies on $C[u, v], w \in X \cap V(C)$ is an endpoint of $e$, and $u$ and $v$ cross at $w$. Suppose that the edges of $C[u, v]$ incident to $w$ are $y^{\prime} w$ and $w y^{\prime \prime}$, so that $u y^{\prime \prime}$ and $v y^{\prime}$ are the two edges forming $u$ and $v$ 's crossing on $w$. Without loss of generality, $e=y^{\prime} w$. The condition that $P$ and $C$ agree on $e$ guarantees that $P[u, w]$ contains $y^{\prime}$.

There are two cases to consider: either both $y^{\prime} w$ and $w y^{\prime \prime}$ are edges of $P$, or just $y^{\prime} w$.

In the first case, let $C^{\prime}:=u P\left[u, y^{\prime}\right] y^{\prime} v P\left[v, y^{\prime \prime}\right] y^{\prime \prime} u$. Then $V\left(C^{\prime}\right) \supseteq V(C)-\{w\}+\{x\}$. If we have strict containment, then $\left|C^{\prime}\right|>|C|$, a contradiction. So we may assume $V\left(C^{\prime}\right) \cap X=(V(C) \cap X)-$ $\{w\}+\{x\}$. Observe that $C^{\prime}$ satisfies Lemma 3.6 for $x^{\prime}=w$. So $D$ is 2-rich. Let $a$ be the vertex in $P$ preceding $z$ and $a^{\prime}$ the vertex in $P$ succeeding $z^{\prime}$ (so $a, a^{\prime} \in V(C)$ ). Let $P^{\prime}$ be a $a, a^{\prime}$-path internally disjoint from $C$ that contains at least 2 internal vertices in $X$. Let $C^{\prime \prime}$ be obtained by replacing in $C^{\prime}$ the segment $P\left[a, a^{\prime}\right]$ with $P^{\prime}$. We have $\left|V\left(C^{\prime \prime}\right) \cap X\right|>|X+\{x\}-\{w\}|$. Therefore $\left|C^{\prime \prime}\right|>|C|$, a contradiction.

In the second case, the cycle $u P\left[u, y^{\prime}\right] y^{\prime} v P[v, w] w y^{\prime \prime} u$ is longer than $C$, since it contains all of $X \cap V(C)$ as well as $x$, a contradiction.

\section{Handling the case $\widetilde{t}=3$}

\subsection{Short, medium, and long-type configurations}

We continue to study properties of a best triple $(C, x, F)$. Recall that by Lemma $3.3, \widetilde{t}=|\widetilde{T}|=3$, so we will assume that $N(D) \cap V(C)=\left\{u_{1}, u_{2}, u_{3}\right\}$. Partition $V(C)-\left\{u_{1}, u_{2}, u_{3}\right\}$ into $U_{1}, U_{2}$ and $U_{3}$, where for $i \in[3], U_{i}=V\left(C\left[u_{i}, u_{i+1}\right]\right)-\left\{u_{i}, u_{i+1}\right\}$, i.e. $U_{i}$ is the set of vertices on $C$ from $u_{i}$ to

$u_{i+1}$ not including either endpoint. Here and in the remainder of the paper, we let the indices on $D$ 's neighbors wrap around modulo 3 , so that, for example, $u_{0}=u_{3}$ and $u_{4}=u_{1}$.

Let $X_{i}=U_{i} \cap X$ and $Y_{i}=U_{i} \cap Y$. For $j>0$, let $x_{i, j}$ be the $j^{\text {th }}$ vertex in $X_{i}$ clockwise; let $x_{i,-j}$ be the $j^{\text {th }}$ vertex in $X_{i-1}$ counterclockwise. For example, $x_{i, 1}=x^{+}\left(u_{i}\right)$ and $x_{i,-1}=x^{-}\left(u_{i}\right)$. Define $y_{i, j}$ similarly.

One of the lines of attack in this section is trying to find a 4-element good subset of $X^{+}(T) \cup X^{-}(T)$, which will contradict Lemma 3.1. This will not work if several of these vertices have many CONs. We will classify the obstacles to this approach into three types. For each $i \in[3]$, we say that:

- $i$ has short type if $x_{i,-1}$ and $x_{i, 1}$ have a CON.

- $i$ has medium type if $x_{i, 1}$ and $x_{i+1,-1}$ have a CON.

- $i$ has long type if $x_{i,-1}$ and $x_{i+1,1}$ have a CON.

These three configurations are shown in Figure 3.

We first prove that each segment $U_{i}$ contains at least two vertices in $X$.

Lemma 4.1. For any $x^{\prime} \in X^{+}(T), d_{C}(x)+d_{C}\left(x^{\prime}\right) \geq 8$. In particular, $d_{C}\left(x^{\prime}\right) \geq 5$. 

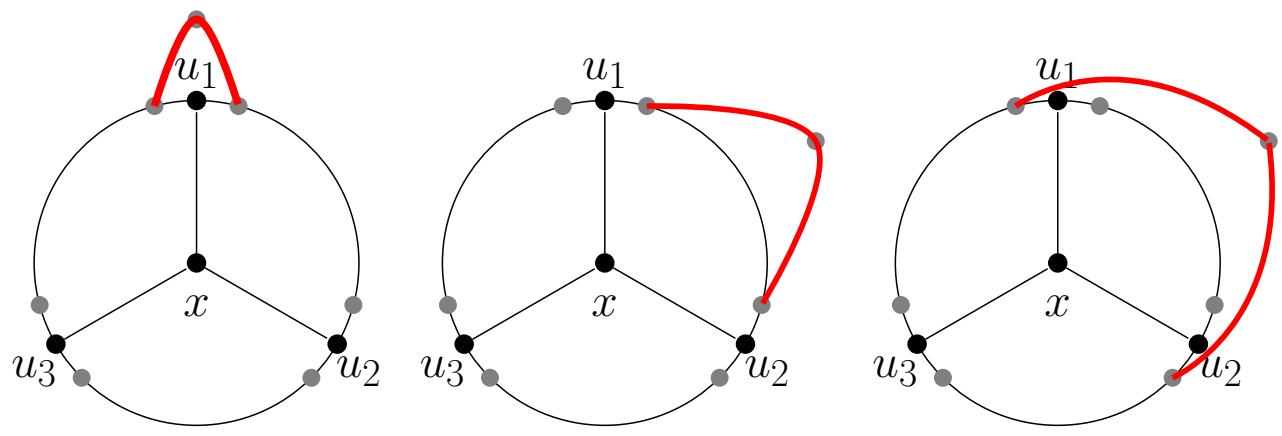

Figure 3: Short-type, medium-type, and long-type configurations.

Proof. Suppose $x^{\prime}=x_{1,1}$ and $d_{C}(x)+d_{C}\left(x^{\prime}\right) \leq 7$. No two vertices in the set $X^{+}(T) \cup\{x\}$ have a CON or cross in $C$. By Lemmas 3.7 and $2.8, d_{C}\left(x_{2,1}\right)+d_{C}\left(x_{3,1}\right) \leq \ell+2$. Therefore

$$
|Y| \geq 4 \delta-\left(d_{C}(x)+d_{C}\left(x_{1,1}\right)\right)-\left(d_{C}\left(x_{2,1}\right)+d_{C}\left(x_{3,1}\right)\right)+\ell \geq 4 \delta-7-(\ell+2)+\ell=4 \delta-9 .
$$

This contradiction proves $d_{C}(x)+d_{C}\left(x^{\prime}\right) \geq 8$. Since $d_{C}(x) \leq t=3, d_{C}\left(x^{\prime}\right) \geq 5$.

Lemma 4.2. For each $i \in[3], x_{i, 1} \neq x_{i+1,-1}$.

Proof. Let $C^{\prime}=u_{i} F\left[u_{i}, u_{i+1}\right] u_{i+1} C\left[u_{i+1}, u_{i}\right] u_{i}$. Then $\left|C^{\prime}\right| \geq|C|$. If the component $D$ of $G-C$ containing $x$ is 2-rich, then $\left|C^{\prime}\right|>|C|$. So by Lemma 3.5, $|T \cap Y| \leq 1$, and hence $d_{C}(x) \leq 1$. By the choice of $(C, x, F)$ as a best triple, $d_{C^{\prime}}\left(x_{i, 1}\right) \leq 1$ as well. Since $V(C)-\left\{y^{-}\left(x_{i, 1}\right), y^{+}\left(x_{i, 1}\right), x_{i, 1}\right\} \subseteq$ $V\left(C^{\prime}\right), N_{C}\left(x_{i, 1}\right) \subseteq N_{C^{\prime}}\left(x_{i, 1}\right) \cup\left\{y^{-}\left(x_{i, 1}\right), y^{+}\left(x_{i, 1}\right)\right\}$, and therefore $d_{C}\left(x_{i, 1}\right) \leq 1+2$. This contradicts Lemma 4.1 .

It is possible that some segments $U_{i}$ contain only two vertices of $X$, but in that case, we can deduce some additional structure we will use later.

Lemma 4.3. For each $i \in[3]$, if $x_{i, 2}=x_{i+1,-1}$, then $i+1$ does not have short or long type.

Proof. If $i+1$ has short or long type, we can find a cycle $C^{\prime}$ such that $X \cap C^{\prime}$ includes $x$ but leaves out $x_{i, 1}$.

If $i+1$ has short type and $y$ is a CON of $x_{i+1,-1}$ and $x_{i+1,1}$, then

$$
C^{\prime}:=u_{i+1} C^{-}\left[u_{i+1}, x_{i+1,-1}\right] x_{i+1,-1} y x_{i+1,1} C\left[x_{i+1,1}, u_{i}\right] u_{i} F\left[u_{i}, u_{i+1}\right] u_{i+1} .
$$

Note that $C$ includes at most three vertices of $Y$ which are not in $C^{\prime}: y^{+}\left(x_{i, 1}\right)$, possibly $y^{-}\left(x_{i, 1}\right)$ (if $u_{i} \in X$ ), and possibly $y^{+}\left(u_{i+1}\right)$ (if $\left.u_{i+1} \in X\right)$.

If $i+1$ has long type and $y$ is a CON of $x_{i+1,-1}$ and $x_{i-1,1}$, then

$$
C^{\prime}:=x_{i+1,-1} C\left[x_{i+1,-1}, u_{i-1}\right] u_{i-1} F\left[u_{i-1}, u_{i}\right] u_{i} C^{-}\left[u_{i}, x_{i-1,1}\right] x_{i-1,1} y x_{i+1,-1} .
$$

Again, $C$ includes at most three vertices of $Y$ which are not in $C^{\prime}: y^{+}\left(x_{i, 1}\right)$, possibly $y^{-}\left(x_{i, 1}\right)$ (if $\left.u_{i} \in X\right)$, and possibly $y^{+}\left(u_{i-1}\right)$ (if $\left.u_{i-1} \in X\right)$. 
In both cases, $\left|C^{\prime}\right| \geq|C|$, with strict inequality if $D$ is 2-rich. So we may assume $D$ is not 2-rich. By Lemma 3.5, $|T \cap Y| \leq 1$, and hence $d_{C}(x) \leq 1$. Therefore by the choice of $(C, x, F)$ as a best triple, $d_{C^{\prime}}\left(x_{i, 1}\right) \leq 1$. Then in either case $d_{C}\left(x_{i, 1}\right) \leq 1+3$, contradicting Lemma 4.1 .

Lemma 4.4. For each $i \in[3]$, one of the following configurations must appear:

(i) $i$ has short type, or

(ii) one of $i-1$ or $i$ has medium type, or

(iii) $i+1$ has long type.

Proof. Suppose for some $i \in[3]$ none of (i)-(iii) holds. Let $W=\left\{x_{i-1,1}, x_{i,-1}, x_{i, 1}, x_{i+1,-1}\right\}$. By Lemma 3.7 (applied to $C$ and also to the backward orientation of $C$ ), the vertices inside the sets $W_{1}=\left\{x_{i-1,1}, x_{i, 1}\right\}$ and $W_{2}=\left\{x_{i,-1}, x_{i+1,-1}\right\}$ have no crossings. By Lemma 2.5. no vertex in $W$ can have a CON with $x$. Since by Lemma 3.1, $W$ is not a good set, some two vertices in $W$ have a CON. By Lemma 2.5 again, $x_{i-1,1}$ and $x_{i, 1}$ have no CONs, and $x_{i,-1}$ and $x_{i+1,-1}$ have no CONs. This leaves the configurations in the statement of this lemma.

The plan of the remainder of this paper is as follows:

1. In the next subsection we define abundant indices and show that not all $i \in[3]$ are abundant. This will help to handle medium-type and short-type configurations.

2. In Subsection 4.3 we show that at most one $i \in[3]$ has long type.

3. In Subsection 4.4 we prove that no $i \in[3]$ has medium type. An important part of this proof is Lemma 4.5 from Subsection 4.2 .

4. In Subsection 4.5 we show that none of $i \in[3]$ has long type. So, by Lemma 4.4. every $i \in[3]$ has short type.

5. Subsection 4.6 finishes the proof of the main theorem by handling the case that every $i \in[3]$ has short type.

\subsection{On abundant indices}

Call an $i \in[3]$ abundant if each of the vertices $x_{i, 2}, x_{i, 3}, \ldots, x_{i+1,-2}$ has a CON with $x_{i, 1}$ and a CON with $x_{i+1,-1}$.

Lemma 4.5. At least one $i \in[3]$ is not abundant.

Proof. Suppose all $i \in[3]$ are abundant. For $i \in[3]$, let $w_{i}=y^{+}\left(x^{-}\left(u_{i}\right)\right)$. In other words, $w_{i}=u_{i}$ if $u_{i} \in Y$, and $w_{i}=y_{i,-1}$ if $u_{i} \in X$. Define $W=\left\{w_{1}, y^{-}\left(w_{1}\right), w_{2}, y^{-}\left(w_{2}\right), w_{3}, y^{-}\left(w_{3}\right)\right\}$. We claim that for all $i \in[3]$,

$$
N_{C}\left(x_{i, 1}\right) \subseteq Y_{i} \cup W
$$

Suppose that $x_{i, 1}$ has a neighbor $y_{j, k}$ where $j \neq i$ and $y_{j, k} \in Y_{j}-\left\{w_{j+1}, y^{-}\left(w_{j+1}\right)\right\}$. By Lemma 2.2 . if $u_{j} \in X$, then $y_{j, k} \neq y_{j, 1}$. So $y_{j, k}$ lies strictly between $x_{j, 1}$ and $x_{j+1,-2}$. Since $j$ is abundant, 


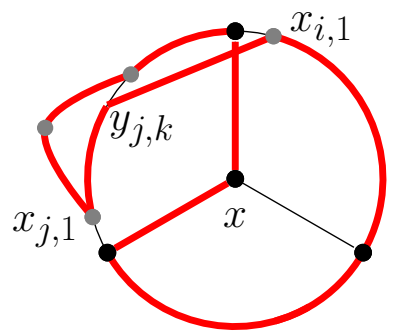

Figure 4: A longer cycle when $x_{i, 1}$ has a neighbor $y_{j, k}$.

$x^{+}\left(y_{j, k}\right)$ and $x_{j, 1}$ have a CON, say $y$. Then the cycle

$$
C^{\prime}:=x_{i, 1} C\left[x_{i, 1}, u_{j}\right] u_{j} F\left[u_{j}, u_{i}\right] u_{i} C^{-}\left[u_{i}, x^{+}\left(y_{j, k}\right)\right] x^{+}\left(y_{j, k}\right) y x_{j, 1} C\left[x_{j, 1}, y_{j, k}\right] y_{j, k} x_{i_{1}}
$$

(see Figure 4) is longer than $C$, a contradiction. This proves (3).

Next we show that

$$
\text { if }\left|X_{j}\right|=2 \text { and } x_{i, 1} y^{+}\left(x_{j, 1}\right) \in E(G) \text {, then } N\left(x_{j, 1}\right) \cap W=\left\{y^{+}\left(x_{j, 1}\right)\right\} \text {. }
$$

Indeed, let $P_{1}$ be a longest $u_{j}, u_{i}$-path all internal vertices of which are in $D=D(C, x)$. Consider the cycle

$$
C^{\prime \prime}:=x_{i, 1} C\left[x_{i, 1}, u_{j}\right] u_{j} P_{1} u_{i} C^{-}\left[u_{i}, y^{+}\left(x_{j, 1}\right)\right] y^{+}\left(x_{j, 1}\right) x_{i_{1}} .
$$

If $D$ is 2-rich, then $C^{\prime \prime}$ is longer than $C$, a contradiction. Thus $D$ is not 2-rich, and hence by Lemma 3.5, $|Y \cap T| \leq 1$. In this case, $\left|C^{\prime \prime}\right| \geq|C|$. Let $F^{\prime \prime}$ be a best $x_{j, 1}, C^{\prime \prime}$-fan. Since the triple $\left(C^{\prime \prime}, x_{j, 1}, F^{\prime \prime}\right)$ is not better than $(C, x, F),\left|C^{\prime \prime}\right|=|C|$ and $\left|N\left(x_{j, 1}\right) \cap V\left(C^{\prime \prime}\right)\right| \leq 1$. Since $y^{+}\left(x_{j, 1}\right) \in N\left(x_{j, 1}\right)$ by definition, and $W \subseteq V\left(C^{\prime \prime}\right)$, (4) follows.

Now we show that similarly to (4),

$$
\text { if }\left|X_{j}\right| \geq 3 \text { and } x_{i, 1} y^{-}\left(w_{j+1}\right) \in E(G) \text {, then }\left|N\left(x_{j, 1}\right) \cap W\right| \leq 1 \text {. }
$$

Indeed, let $P_{1}$ be a longest $u_{j}, u_{i}$-path all internal vertices of which are in $D=D(C, x)$. Since $\left|X_{j}\right| \geq 3$ and $j$ is abundant, $x_{j+1,-1}$ and $x_{j, 2}$ have a CON, say $y$. Consider the cycle

$$
C^{\prime \prime \prime}:=x_{i, 1} C\left[x_{i, 1}, u_{j}\right] u_{j} P_{1} u_{i} C^{-}\left[u_{i}, x_{j+1,-1}\right] x_{j+1,-1} y x_{j, 2} C\left[x_{j, 2}, y^{-}\left(w_{j+1}\right)\right] y^{-}\left(w_{j+1}\right) x_{i_{1}} .
$$

If $D$ is 2-rich, then $C^{\prime \prime \prime}$ is longer than $C$, a contradiction. Thus $D$ is not 2-rich, and by Lemma 3.5 , $|Y \cap T| \leq 1$. In this case, $\left|C^{\prime \prime}\right| \geq|C|$. Let $F^{\prime \prime \prime}$ be a best $x_{j, 1}, C^{\prime \prime \prime}$-fan. Since the triple $\left(C^{\prime \prime \prime}, x_{j, 1}, F^{\prime \prime \prime}\right)$ is not better than $(C, x, F),\left|C^{\prime \prime \prime}\right|=|C|$ and $\left|N\left(x_{j, 1}\right) \cap V\left(C^{\prime \prime \prime}\right)\right| \leq 1$. Since $W \subseteq V\left(C^{\prime \prime \prime}\right)$, (5) follows.

If there are no distinct $i, j \in[3]$ such that $x_{i, 1} y^{-}\left(w_{j+1}\right) \in E(G)$, then by $(3), \sum_{i \in[3]}\left|N_{C}\left(x_{i, 1}\right)\right| \leq$ $\sum_{i \in[3]}\left(\left|Y_{i}\right|+2\right)$, and hence

$$
\sum_{i \in[3]} N_{C}\left(x_{i, 1}\right) \leq \ell+6 .
$$

If there is only one $j \in[3]$ such that $y^{-}\left(w_{j+1}\right)$ is adjacent to $x_{j-1,1}$ or to $x_{j+1,1}$ (say, $x_{i, 1} y^{-}\left(w_{j+1}\right) \in$ $E(G)$ ), then by (3), $\left|N_{C}\left(x_{i, 1}\right)\right| \leq\left|Y_{i}\right|+3$ for $i \neq j$, but by (4) and (5), $\left|N_{C}\left(x_{j, 1}\right)\right| \leq\left|Y_{j}\right|$. So 
again (6) holds.

Finally, if there are distinct $j_{1}, j_{2} \in[3]$ such that $x_{i_{s}, 1} y^{-}\left(w_{j_{s}+1}\right) \in E(G)$ for $s \in[2]$ and some $i_{s}$, then by (4) and (5), $\left|N_{C}\left(x_{j_{s}, 1}\right)\right| \leq\left|Y_{j_{s}}\right|$, and by (3), $\left|N_{C}\left(x_{i, 1}\right)\right| \leq\left|Y_{i}\right|+4$ for $i \in[3]-\left\{j_{1}, j_{2}\right\}$. Thus (6) holds in all cases.

By Lemma 2.5, no two vertices in the set $A=\left\{x, x_{1,1}, x_{2,1}, x_{3,1}\right\}$ have a CON. Therefore, by (6), $|Y| \geq \ell+4 \delta-3-(\ell-6)=4 \delta-9$, a contradiction.

\subsection{Eliminating multiple long-type configurations}

Lemma 4.6. At most one $i \in[3]$ has long type.

Proof. Suppose the lemma does not hold. By symmetry, we may assume that $x_{3,-1}$ and $x_{1,1}$ have a CON $a$, and $x_{1,-1}$ and $x_{2,1}$ have a CON $b$. Since $x_{1,1}$ and $x_{2,1}$ cannot have a CON, $a \neq b$. Consider the cycle

$$
C^{\prime}:=u_{3} C\left[u_{3}, x_{1,-1}\right] x_{1,-1} b x_{2,1} C\left[x_{2,1}, x_{3,-1}\right] x_{3,-1} a x_{1,1} C\left[x_{1,1}, u_{2}\right] u_{2} F\left[u_{2}, u_{3}\right] u_{3}
$$

formed as shown in Figure 5 .

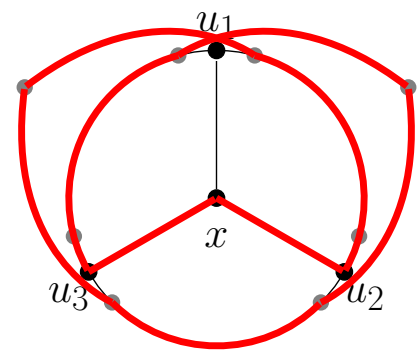

Figure 5: The cycle $C^{\prime}$ formed by two long-type configurations.

Cycle $C^{\prime}$ includes $x$ and all vertices of $X \cap V(C)$, except possibly $u_{1}$, hence $\left|C^{\prime}\right| \geq|C|$. If $u_{1} \in Y$, $C^{\prime}$ is longer than $C$, which is a contradiction. Moreover, if $F\left[u_{2}, u_{3}\right]$ contains at least 2 internal $X$ vertices, then $\left|C^{\prime}\right|>|C|$.

If $u_{1} \in X$, let $y u_{1}$ be the last edge of the $x, u_{1}$-path of $F$. As $G$ is 3 -connected, there is a path $P$ from $y$ to $V(C) \cap V\left(C^{\prime}\right)$ not containing $x$ or $u_{1}$. Since by definition, deleting $\left\{u_{1}, u_{2}, u_{3}\right\}$ disconnects $x$, and therefore $y$, from $C$, path $P$ must go from $y$ to some vertex $u^{\prime}$ on either the $x, u_{2}$-path or the $x, u_{3}$-path in $F$. Without loss of generality, assume $u^{\prime}$ is on the $x, u_{2}$-path.

Consider the cycle

$$
C^{\prime \prime}:=u_{2} C^{-}\left[u_{2}, x_{1,1}\right] x_{1,1} a x_{3,-1} C^{-}\left[x_{3,-1}, x_{2,1}\right] x_{2,1} b x_{1,-1} C^{-}\left[x_{1,-1}, u_{3}\right] u_{3} F\left[u_{3}, y\right] y P u^{\prime} F\left[u^{\prime}, u_{2}\right] u_{2}
$$

shown in Figure 6, obtained from $C^{\prime}$ by replacing the segment $C^{\prime}\left[u^{\prime}, x\right]$ contained in $F$ by the union of $P$ and $F[x, y]$. This is longer than $C^{\prime}$ (and therefore longer than $C$ ) except in one case: when 
each of $P$ and the $F[x, y]$ is a single edge, and $u^{\prime}=u_{2}$ (which must then be in $X$ ). In this case,

$$
C^{\prime \prime}:=u_{2} C^{-}\left[u_{2}, x_{1,1}\right] x_{1,1} a x_{3,-1} C^{-}\left[x_{3,-1}, x_{2,1}\right] x_{2,1} b x_{1,-1} C^{-}\left[x_{1,-1}, u_{3}\right] u_{3} F\left[u_{3}, x\right] x y u_{2} .
$$

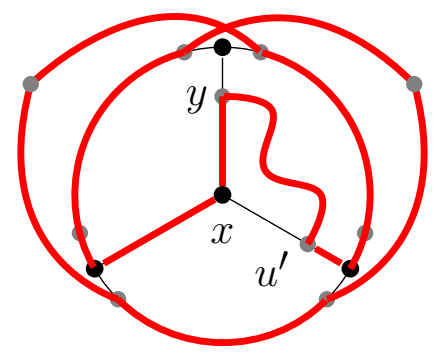

Figure 6: The cycle $C^{\prime \prime}$ formed using the path $P$.

Let $F^{\prime \prime}$ be the $u_{1}, C^{\prime \prime}$-fan formed by the paths $C\left[x_{1,-1}, u_{1}\right]$ and $C\left[u_{1}, x_{1,1}\right]$, and the edge $u_{1} y$. The triple $\left(C^{\prime \prime}, u_{1}, F^{\prime \prime}\right)$ has $\left|C^{\prime \prime}\right|=|C|$ and $t\left(u_{1}, C^{\prime \prime}\right)=t(x, C)$, so by our choice of the triple $(C, x, F)$, we must have $\left|V\left(F^{\prime \prime}\right) \cap V\left(C^{\prime \prime}\right) \cap Y\right| \leq|V(F) \cap V(C) \cap Y|$. Since $V\left(F^{\prime \prime}\right) \cap V\left(C^{\prime \prime}\right) \cap Y=\{y\}$, $|V(F) \cap V(C) \cap Y| \geq 1$, which can only happen if $u_{3} \in Y$. Therefore the $x, u_{3}$-path in $F$ consists of a single edge $x u_{3}$, and the only vertices of $V\left(C^{\prime \prime}\right)-V(C)$ are $x, y, a$, and $b$.

Let $y^{\prime}$ be the vertex of $F$ between $x$ and $u_{2}$ on the $x, u_{2}$-path of $F$. Since $G$ is 3 -connected, there is a path $P^{\prime}$ from $y^{\prime}$ to $V(C) \cup V\left(C^{\prime \prime}\right)$ not containing $x$ or $u_{2}$. However, we know that deleting $\left\{u_{1}, u_{2}, u_{3}\right\}$ disconnects $x$, and therefore $y^{\prime}$, from $C$. Therefore either $P^{\prime}$ goes from $y^{\prime}$ to a vertex in $V\left(C^{\prime \prime}\right)-V(C)$, which can only be $y$, or else $P^{\prime}$ goes from $y^{\prime}$ to one of the vertices $u_{1}, u_{3}$.
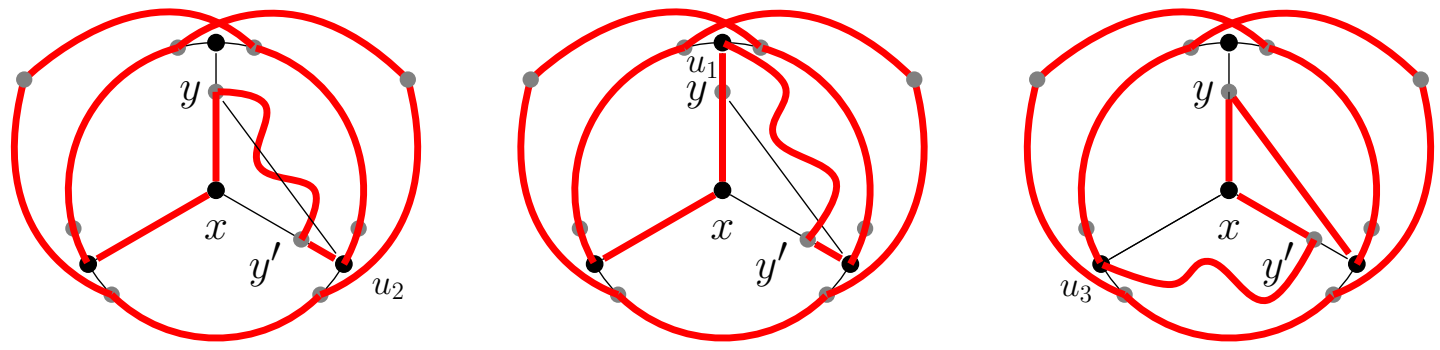

Figure 7: Three ways to extend $C^{\prime \prime}$ to a longer cycle

In each of these cases, we obtain a longer cycle. If $P^{\prime}$ goes from $y^{\prime}$ to $y$, we can extend $C^{\prime \prime}$ by replacing edge $u_{2} y$ with $u_{2} y^{\prime}$ followed by $P^{\prime}$ to get the cycle

$$
u_{2} C^{-}\left[u_{2}, x_{1,1}\right] x_{1,1} a x_{3,-1} C^{-}\left[x_{3,-1}, x_{2,1}\right] x_{2,1} b x_{1,-1} C^{-}\left[x_{1,-1}, u_{3}\right] u_{3} F\left[u_{3}, x\right] x y P^{\prime} y^{\prime} u_{2},
$$

as shown on the left in Figure 7. If $P^{\prime}$ goes from $y^{\prime}$ to $u_{1}$, we can extend $C^{\prime \prime}$ by replacing edge $u_{2} y$ with $u_{2} y^{\prime}, P^{\prime}$, and $u_{1} y$ to get the cycle

$$
u_{2} C^{-}\left[u_{2}, x_{1,1}\right] x_{1,1} a x_{3,-1} C^{-}\left[x_{3,-1}, x_{2,1}\right] x_{2,1} b x_{1,-1} C^{-}\left[x_{1,-1}, u_{3}\right] u_{3} F\left[u_{3}, u_{1}\right] u_{1} P^{\prime} y^{\prime} u_{2},
$$


as shown in the middle of Figure 7. Finally, if $P^{\prime}$ goes from $y^{\prime}$ to $u_{3}$, we can extend $C^{\prime \prime}$ by replacing edge $x u_{3}$ with $x y^{\prime}$ followed by $P^{\prime}$ to get the cycle

$$
u_{2} C^{-}\left[u_{2}, x_{1,1}\right] x_{1,1} a x_{3,-1} C^{-}\left[x_{3,-1}, x_{2,1}\right] x_{2,1} b x_{1,-1} C^{-}\left[x_{1,-1}, u_{3}\right] u_{3} P^{\prime} y^{\prime} F\left[y^{\prime}, y\right] y u_{2},
$$

as shown on the right in Figure 7.

Thus, no more than one $i \in[3]$ can have long type.

\subsection{Eliminating medium-type configurations}

In this subsection, our goal is to show that no $i \in[3]$ has medium type.

Recall that $i \in[3]$ is abundant if each of the vertices $x_{i, 2}, x_{i, 3}, \ldots, x_{i+1,-2}$ has a CON with $x_{i, 1}$ and a CON with $x_{i+1,-1}$.

Lemma 4.7. If $i \in[3]$ has medium type, then $i$ is abundant.

Proof. Without loss of generality, we will assume that $i=1$ has medium type. We will show that for all $j \geq 1, x_{2,-1}$ and $x_{2,-j}$ share a CON. This is the same as showing $x_{2,-1}$ and $x_{1, a}$ share a CON for all $a \geq 1$ such that $x_{1, a} \neq x_{2,-1}$. Showing that $x_{1,1}$ and $x_{1, j}$ have a CON is symmetric.

Suppose there is an $a$ such that $x_{1, a}$ shares no CON with $x_{2,-1}$, but $x_{1, a^{\prime}}$ does for all $1 \leq a^{\prime}<a$. Our goal is to show $\left\{x_{1,-1}, x_{2,-1}, x_{3,-1}, x_{1, a}\right\}$ is a good set. Let $y^{\prime}$ be the common neighbor of $x_{2,-1}$ and $x_{1, a-1}$. Note that $x_{1,-1}, x_{2,-1}, x_{3,-1}$ can have no CON by Lemma 2.5. Additionally, by Lemma 3.7, $x_{2,-1}, x_{3,-1}$ have no crossings.

By our choice of $a$, vertices $x_{1, a}$ and $x_{2,-1}$ have no CON. By Lemma 3.8 via the path

$$
P:=x_{1, a} C\left[x_{1, a}, x_{2,-1}\right] x_{2,-1} y^{\prime} x_{1, a-1} C^{-}\left[x_{1, a-1}, u_{1}\right] u_{1} F\left[u_{1}, u_{2}\right] u_{2} C\left[u_{2}, x_{1,-1}\right] x_{1,-1}
$$

shown in Figure 8, $x_{1, a}$ and $x_{1,-1}$ have no crossings and no CON outside $P$. However, $y^{\prime}$ is the only possible CON of $x_{1, a}$ and $x_{1,-1}$ on $P$, and if $x_{1,-1} y^{\prime} \in E(G), x_{1,-1}$ and $x_{2,-1}$ would have a CON, which also is impossible.

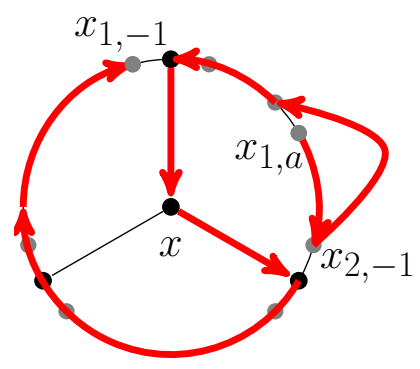

Figure 8: The path $P$ from $x_{1, a}$ to $x_{1,-1}$.

Finally, we argue $x_{1, a}$ and $x_{3,-1}$ have no CON. Suppose $y$ is such a CON; then the cycle

$$
u_{3} C\left[u_{3}, x_{1, a-1}\right] x_{1, a-1} y^{\prime} x_{2,-1} C^{-}\left[x_{2,-1}, x_{1, a}\right] x_{1, a} y x_{3,-1} C^{-}\left[x_{3,-1}, u_{2}\right] u_{2} F\left[u_{2}, u_{3}\right] u_{3}
$$


is a longer cycle than $C$. So $x_{1, a}$ has no CONs with any of $x_{1,-1}, x_{2,-1}, x_{3,-1} ; x_{1, a}$ and $x_{1-1}$ have no crossings, and neither do $x_{2,-1}$ and $x_{3,-1}$. This certifies that $\left\{x_{1,-1}, x_{2,-1}, x_{3,-1}, x_{1, a}\right\}$ is a good set, a contradiction to Lemma 3.1 .

Lemma 4.8. If $i$ has medium type, then for $x_{i, j} \in\left\{x_{i, 1}, \ldots, x_{i+1,-2}\right\}$,

(i) $x_{i, j}$ and $x_{i+1,1}$ have no CONs and no crossings, and

(ii) $x_{i, j}$ and $x_{i-1,1}$ have no CONs.

Symmetrically, $x_{i, j} \in\left\{x_{i, 2}, \ldots, x_{i+1,-1}\right\}$ and $x_{i,-1}$ have no CONs and no crossings, and $x_{i, j}$ and $x_{i-1,-1}$ have no CONs.

Proof. Without loss of generality, let $i=1$. Suppose $x_{1, j}$ and $x_{2,1}$ have a common neighbor $y$ (the $x_{1,-1}$ case is symmetric). By Lemma 4.7, $x_{1,1}$ and $x_{1, j+1}$ have a CON $y^{\prime}$. By Lemma 3.8 and the path

$$
P:=x_{1, j} C^{-}\left[x_{1, j}, x_{1,1}\right] x_{1,1} y^{\prime} x_{1, j+1} C\left[x_{1, j+1}, u_{2}\right] u_{2} F\left[u_{2}, u_{1}\right] u_{1} C^{-}\left[u_{1}, x_{2,1}\right] x_{2,1},
$$

shown in Figure 9, $x_{1, j}$ and $x_{2,1}$ share no CONs (otherwise $x_{1,1}$ and $x_{2,1}$ share a CON) and no crossings.

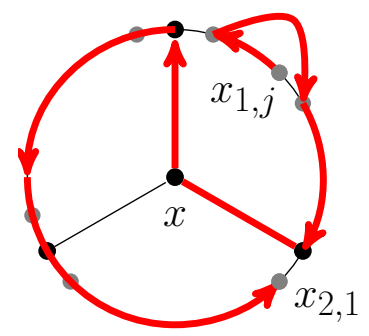

Figure 9: The path $P$ from $x_{1, j}$ to $x_{2,1}$.

Suppose that $x_{1, j}$ has a CON $y$ with $x_{3,1}$. By Lemma 4.7, $x_{1,1}$ and $x_{1, j+1}$ have a CON $y^{\prime}$. Moreover, by Lemma 2.5, $x_{1,1}$ and $x_{3,1}$ can have no CON, so $y \neq y^{\prime}$. In this case, we obtain a longer cycle than $C$ : the cycle

$$
x_{1,1} C\left[x_{1,1}, x_{1, j}\right] x_{1, j} y x_{3,1} C\left[x_{3,1}, u_{1}\right] u_{1} F\left[u_{1} u_{3}\right] u_{3} C^{-}\left[u_{3}, x_{1, j+1}\right] x_{1, j+1} y^{\prime} x_{1,1} .
$$

This is a contradiction, so $x_{1, j}$ and $x_{3,1}$ have no CON. The $x_{3,-1}$ case is symmetric.

Lemma 4.9. If $j \in[3]$ does not have medium type, then every $i \in[3]$ that has medium type also has long type.

Proof. Without loss of generality, suppose $i$ has medium type but $j=i-1$ does not. The case where $j=i+1$ is symmetric, after reorienting $C$. It suffices to show that in such a case, $i$ has long type.

By Lemma 4.2, we may assume $x_{i, 1} \neq x_{i+1,-1}$. Let $A=\left\{x_{i, 2}, x_{i+1,1}, x_{i,-1}, x_{i-1,1}\right\}$. By Lemma 4.8 $x_{i,-1}$ and $x_{i, 2}$ have no CONs or crossings; by Lemma 2.5 and Lemma 3.7, $x_{i-1,1}$ and $x_{i+1,1}$ have no CONs or crossings. 
If $x_{i, 2} \neq x_{i+1,-1}$, then Lemma 4.8 further tells us that $x_{i, 2}$ has no CONs with $x_{i-1,1}$ or $x_{i+1,1}$. If $x_{i, 2}=x_{i+1,-1}$ then Lemma 4.3 gives the same conclusion.

By assumption, $i-1$ does not have medium type, so $x_{i-1,1}$ and $x_{i,-1}$ have no CONs. If $x_{i,-1}$ and $x_{i+1,1}$ also have no CONs, then $A$ is a good set, contradicting Lemma 3.1. Therefore $x_{i,-1}$ and $x_{i+1,1}$ must have a CON; in other words, $i$ has long type.

The three previous lemmas help us to prove the main result of this subsection:

Lemma 4.10. No $i \in[3]$ has medium type.

Proof. Suppose the lemma does not hold. If all $i \in[3]$ have medium type, then by Lemma 4.7, all of them are abundant, a contradiction to Lemma 4.5. Thus there is a $j \in[3]$ that does not have medium type. Then by Lemma 4.9, each $i \in[3]$ that has medium type also has long type. Now Lemma 4.6 yields that only one $i$ can have medium type. Suppose by symmetry that this $i$ is 1 .

Let $b$ be the smallest integer such that $x_{2,1}$ and $x_{1,-b}$ have no CON, and consider instead the set $X^{\prime}=\left\{x_{1,2}, x_{2,1}, x_{3,-1}, x_{1,-b}\right\}$. Let $y$ be the CON of $x_{1,-b+1}$ and $x_{2,1}$. By Lemma 3.8 and the path

$$
x_{1,-b} C^{-}\left[x_{1,-b}, u_{3}\right] u_{3} F\left[u_{3}, u_{2}\right] u_{2} C^{-}\left[u_{2}, x_{1,-b+1}\right] x_{1,-b+1} y x_{2,1} C\left[x_{2,1}, x_{3,-1}\right] x_{3,-1}
$$

shown in Figure 10 (left), $x_{3,-1}$ and $x_{1,-b}$ have no CON (otherwise $x_{3,-1}$ and $x_{2,1}$ have a CON, making 2 medium-type) and can only cross at a vertex $x_{1, j}$ for $j \geq 1$ or a vertex $x_{1,-a}$ where $a<b$. Note by Lemma 2.6 they cannot cross at $u_{1}$.

In the first case, if $j>1$, let $y^{-}=y^{-}\left(x_{1, j}\right)$. Note that $x_{2,-1}$ and $x^{-}\left(y^{-}\right)$share a CON $y^{\prime}$. We get a contradiction by the cycle

$$
u_{3} C\left[u_{3}, x^{-}\left(y^{-}\right)\right] x^{-}\left(y^{-}\right) y^{\prime} x_{2,-1} C^{-}\left[x_{2,-1}, y^{-}\right] y^{-} x_{3,-1} C\left[x_{3,-1}, u_{2}\right] u_{2} F\left[u_{2}, u_{3}\right] u_{3} .
$$

If $j=1$, then let $y^{\prime}$ be a CON of $x_{1,1}$ and $x_{1,2}$, and let $y$ be a CON of $x_{2,1}$ and $x_{1,-b+1}$. Then we get the longer cycle

$$
x_{1,1} y^{\prime} x_{1,2} C\left[x_{1,2}, u_{2}\right] u_{2} F\left[u_{2}, u_{1}\right] u_{1} C^{-}\left[u_{1}, x_{1,-b+1}\right] x_{1,-b+1} y x_{2,1} C^{-}\left[x_{2,1}, x_{1,-b}\right] x_{1,-b} y^{+}\left(x_{1,1}\right) x_{1,1} .
$$

In the second case, let $y^{\prime}$ be a CON of $x_{2,1}$ and $x_{1,-a}$. Then we get a longer cycle

$$
u_{3} C\left[u_{3}, y^{-}\left(x_{1,-a}\right)\right] y^{-}\left(x_{1,-a}\right) x_{3,-1} C^{-}\left[x_{3,-1}, x_{2,1}\right] x_{2,1} y^{\prime} x_{1,-a} C\left[x_{1,-a}, u_{2}\right] u_{2} F\left[u_{2}, u_{3}\right] u_{3} \text {. }
$$

By Lemma 4.8, $x_{1,2}$ and $x_{2,1}$ have no CONs and no crossings, and $x_{1,2}$ shares no CONs with $x_{3,-1}$.

Suppose $y^{\prime}$ is a CON of $x_{1,2}$ and $x_{1,-b}$. By the choice of $b, x_{2,1}$ and $x_{1,-b+1}$ have a CON $y$. The cycle

$$
C^{\prime}:=x_{1,-b+1} y x_{2,1} C\left[x_{2,1}, x_{1,-b}\right] x_{1,-b} y^{\prime} x_{1,2} C\left[x_{1,2}, u_{2}\right] u_{2} F\left[u_{2}, u_{1}\right] u_{1} C^{-}\left[u_{1}, x_{1,-b+1}\right] x_{1,-b+1}
$$

shown in Figure 10 (right) excludes $x_{1,1}$ but contains the rest of $X \cap V(C)-\left\{x_{1,1}\right\}$. Moreover, $C^{\prime}$ contains all but at most four vertices in $Y \cap C: y^{+}\left(x_{1,-b}\right), y^{+}\left(x_{1,1}\right)$, and possibly $y^{-}\left(x_{1,1}\right)$ or $y^{-}\left(x_{2,1}\right)$, if $u_{1} \in X$ or $u_{2} \in X$ respectively. If $D$ is 2-rich, then $\left|C^{\prime}\right|>|C|$, so we may assume that $D$ is not 2-rich, and $d_{C}(x) \leq 1$ by Lemma 3.5. By the choice of $(C, x, F)$ as a best triple, $d_{C^{\prime}}\left(x_{1,1}\right) \leq 1$ 

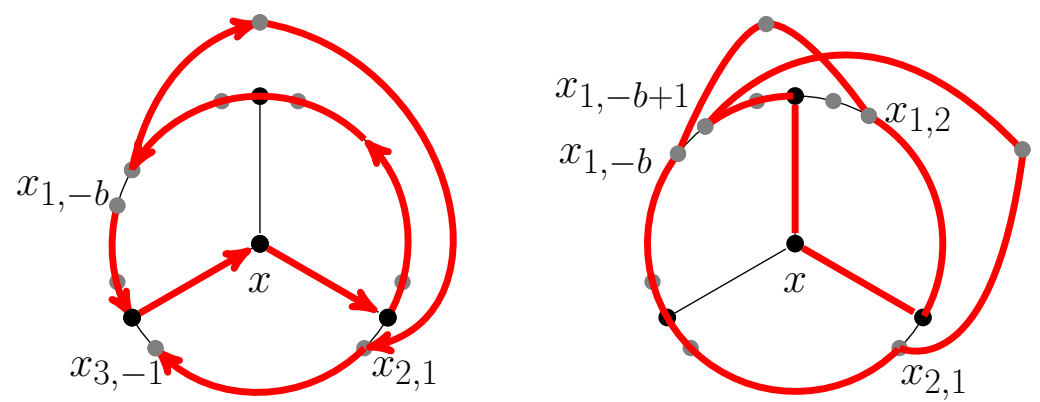

Figure 10: An $x_{1,-b}, x_{3,-1}$-path, and a longer cycle obtained when $x_{1,2}$ and $x_{1,-b}$ have a CON.

as well. Then $d_{C}(x)+d_{C}\left(x_{1,1}\right) \leq 1+1+4$. This contradicts Lemma 4.1, which shows that $x_{1,2}$ and $x_{1,-b}$ share no CONs.

Since 2 does not have medium type, $x_{2,1}$ and $x_{3,-1}$ share no CONs. By the definition of $b, x_{1,-}$ and $x_{2,1}$ share no CONs. Thus, $X^{\prime}$ is good, a contradiction to Lemma 3.1 .

\subsection{Eliminating long-type configurations}

Lemma 4.11. No $i \in[3]$ has long type.

Proof. Suppose some $i \in[3]$ has long type. By Lemma 4.6, there is only one such $i$. By symmetry, assume $x_{3,-1}$ and $x_{1,1}$ have a CON $a$, i.e., only 3 has long type. Then by Lemma 4.4, since no $j$ has medium type, 1 has short type, which means $x_{1,-1}$ and $x_{1,1}$ have a CON $b$.

Let $W=\left\{x_{1,-1}, x_{1,2}, x_{2,1}, x_{3,1}\right\}$. We will show that $W$ is a good set.

By Lemma 2.5 and Lemma 3.7, $x_{2,1}$ and $x_{3,1}$ have no CON or crossings. Also, $x_{1,-1}$ and $x_{1,2}$ have no CON or crossings: This follows from Lemma 3.8, as shown on the left in Figure 11, where the path

$$
P:=x_{1,-1} C^{-}\left[x_{1,-1}, u_{3}\right] u_{3} F\left[u_{3}, u_{1}\right] u_{1} C\left[u_{1}, x_{1,1}\right] x_{1,1} a x_{3,-1} C^{-}\left[x_{3,-1}, x_{1,2}\right] x_{1,2}
$$

agrees with the cycle $C$ on all edges.

We now show that the remaining pairs in $W$ do not have CONs. If $x_{1,-1}$ and $x_{2,1}$ have a CON, then we have a second long-type configuration. If $x_{1,-1}$ and $x_{3,1}$ have a CON, the we have a medium-type configuration.

If $x_{1,2}$ and $x_{2,1}$ have a CON $c$, then the cycle

$$
u_{3} C\left[u_{3}, x_{1,1}\right] x_{1,1} a x_{3,-1} C^{-}\left[x_{3,-1}, x_{2,1}\right] x_{2,1} c x_{1,2} C\left[x_{1,2}, u_{2}\right] u_{2} F\left[u_{2}, u_{3}\right] u_{3}
$$

is longer than $C$, as shown in the middle of Figure 11 . Finally, if $x_{1,2}$ and $x_{3,1}$ have a CON $c$ then the cycle

$$
x_{3,1} C\left[x_{3,1}, x_{1,-1}\right] x_{1,-1} b x_{1,1} C^{-}\left[x_{1,1}, u_{1}\right] u_{1} F\left[u_{1}, u_{3}\right] u_{3} C^{-}\left[u_{3}, x_{1,2}\right] x_{1,2} c x_{3,1}
$$

is longer than $C$, as shown on the right in Figure 11 . 

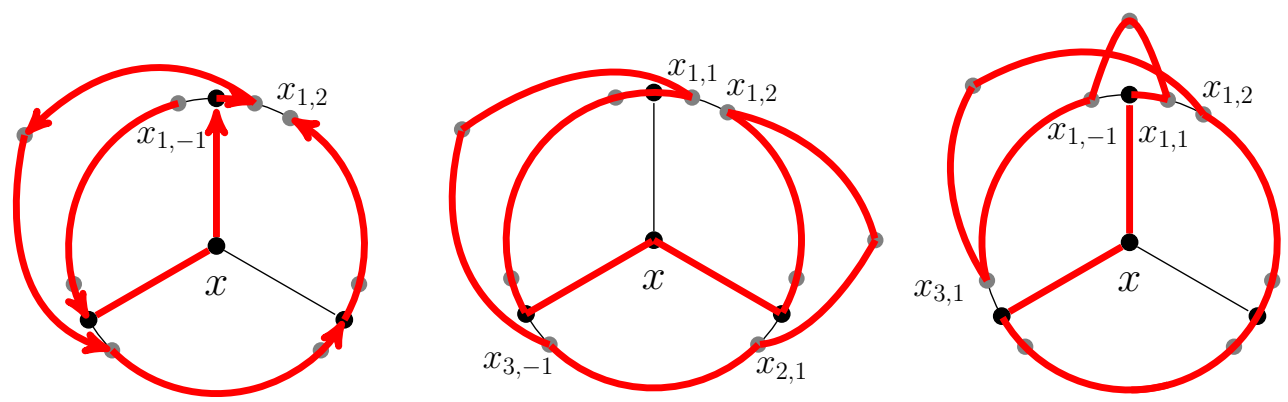

Figure 11: An $x_{1,-1}, x_{1,2}$-path, and longer cycles obtained if $x_{1,2}$ has a CON with $x_{2,1}$ or $x_{3,1}$.

Therefore $W$ is a good set, contradicting Lemma 3.1

\subsection{Eliminating short-type configurations and finishing the proof of Theorem 1.4}

Lemma 4.12. If there are no long-type configurations and no medium-type configurations, then every $i \in[3]$ is abundant.

Proof. By Lemma 4.4, every $i \in[3]$ has short type and no other types.

For definiteness, consider $i=1$. By the definition of short type, $x_{1,-1}$ and $x_{1,1}$ have a CON. Let $b>1$ be the least integer such that $x_{1,-1}$ has no CON with $x_{1, b}$. Some such $b$ exists, because $x_{2,-1}$ has no CON with $x_{1,-1}$. Moreover, if $x_{1, b}=x_{2,-1}$, then we find a cycle $C^{\prime}$ longer than $C$ : if $y_{1}$ is a CON of $x_{1,-1}$ and $x_{2,-2}$, and $y_{2}$ is a CON of $x_{2,-1}$ and $x_{2,1}$, then $y_{1} \neq y_{2}$ (since $x_{1,-1}$ has no CON with $x_{2,-1}$ ) and therefore

$$
u_{1} C\left[u_{1}, x_{2,-2}\right] x_{2,-2} y_{1} x_{1,-1} C^{-}\left[x_{1,-1}, x_{2,1}\right] x_{2,1} y_{2} x_{2,-1} C\left[x_{2,-1}, u_{2}\right] u_{2} F\left[u_{2}, u_{1}\right] u_{1}
$$

is a cycle longer than $C$. So $b$ exists and $x_{1, b} \neq x_{2,-1}$. Note that this implies $x_{1,2} \neq x_{2,-1}$.

Consider the set $W_{b}=\left\{x_{1,-1}, x_{1, b}, x_{2,-1}, x_{3,1}\right\}$. We will show that it is almost a good set.

By Lemma 2.5 and Lemma $3.7, x_{1,-1}$ and $x_{2,-1}$ have no CON or crossing. A CON of $x_{1, b}$ and $x_{3,1}$ is distinct from any CON of $x_{1,-1}$ and $x_{1, b-1}$ because $x_{1,-1}$ and $x_{3,1}$ have no CON. Let $c$ be the CON of $x_{1,-1}$ and $x_{1, b-1}$. By applying Lemma 3.8 to the path

$$
x_{3,1} C\left[x_{3,1}, x_{1,-1}\right] x_{1,-1} c x_{1, b-1} C^{-}\left[x_{1, b-1}, u_{1}\right] u_{1} F\left[u_{1}, u_{3}\right] u_{3} C^{-}\left[u_{3}, x_{1, b}\right] x_{1, b},
$$

as on the left in Figure 12, we see that they can have no other CON, and can only cross at a vertex $x_{1, a}$ with $a<b$.

If such a crossing existed, however, then in particular $x_{3,1}$ would be adjacent to a neighbor of $x_{1, a}$ and letting $c$ be the CON of $x_{1,-1}$ and $x_{1, a+1}$ we would obtain a longer cycle

$$
x_{3,1} C\left[x_{3,1}, x_{1,-1}\right] x_{1,-1} c x_{1, a+1} C\left[x_{1, a+1}, u_{3}\right] u_{3} F\left[u_{3}, u_{1}\right] u_{1} C\left[u_{1}, y^{-}\left(x_{1, a+1}\right)\right] y^{-}\left(x_{1, a+1}\right) x_{3,1}
$$

as shown on the right of Figure 12. In the special case $a=b-1$, the cycle looks only slightly 
different. Letting $c$ be the CON of $x_{1,-1}$ and $x_{1, b-1}$, it is

$$
x_{3,1} C\left[x_{3,1}, x_{1,-1}\right] x_{1,-1} c x_{1, b-1} C^{-}\left[x_{1, b-1}, u_{1}\right] u_{1} F\left[u_{1}, u_{3}\right] u_{3} C^{-}\left[u_{3}, y^{+}\left(x_{1, b-1}\right)\right] y^{+}\left(x_{1, b-1}\right) x_{3,1} \text {. }
$$

We conclude that $x_{1, b}$ and $x_{3,1}$ have no CON or crossings.

By the choice of $b, x_{1,-1}$ and $x_{1, b}$ have no CON. The pair $x_{1,-1}$ and $x_{3,1}$ have no CON, otherwise a medium-type configuration would be formed. The pair $x_{2,-1}$ and $x_{3,1}$ have no CON, otherwise a long-type configuration would be formed.
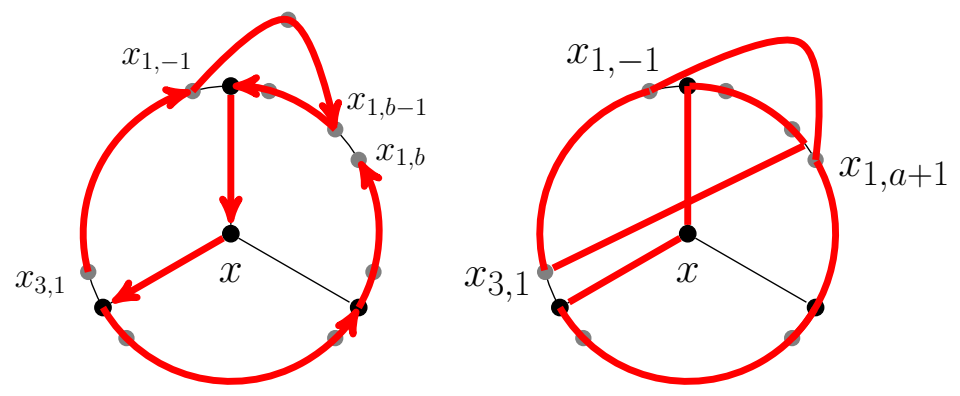

Figure 12: An $x_{3,1}, x_{1, b}$-path, and a longer cycle obtained if $x_{1, b}$ and $x_{3,1}$ have a crossing at $x_{1, a}$.

If $x_{1, b}$ and $x_{2,-1}$ have no CON, then $W_{b}$ is a good set, a contradiction to Lemma 3.1. Thus, $x_{1, b}$ and $x_{2,-1}$ have a CON.

We now prove that

$$
\text { for each } c \geq b \text { such that } x_{1, c} \in C\left[x_{1, b}, x_{2,-2}\right] \text {, vertices } x_{1, c} \text { and } x_{2,-1} \text { have a CON. }
$$

Indeed, suppose (7) does not hold and $c$ is the least integer such that $c \geq b$ and $x_{1, c}$ has no CON with $x_{2,-1}$. By the previous paragraph, $c>b$. Consider the set $W_{c}=\left\{x_{1,-1}, x_{1, c}, x_{2,-1}, x_{3,-1}\right\}$. We will show that this is a good set.

Indeed, $x_{1,-1}$ and $x_{2,-1}$ have no CON or crossing, by Lemma 2.5 and Lemma 3.7. Any CON of $x_{1, c}$ and $x_{3,-1}$ is distinct from any CON of $x_{1, c-1}$ and $x_{2,-1}$, since $x_{3,-1}$ and $x_{2,-1}$ have no CON. They have no other CON or crossings, as shown by the path

$$
x_{1, c} C\left[x_{1, c}, x_{2,-1}\right] x_{2,-1} q x_{1, c-1} C^{-}\left[x_{1, c-1}, u_{3}\right] u_{3} F\left[u_{3}, u_{2}\right] u_{2} C\left[u_{2}, x_{3,-1}\right] x_{3,-1}
$$

(see the left in Figure 13) and Lemma 3.8, where $q$ is the CON of $x_{2,-1}$ and $x_{1, c-1}$.

We show that the remaining pairs have no CONs. Indeed, $x_{1, c}$ and $x_{2,-1}$ have no CON by our choice of $c$. The pairs $\left\{x_{1,-1}, x_{3,-1}\right\}$ and $\left\{x_{2,-1}, x_{3,-1}\right\}$ have no CONs, by Lemma 2.5. Finally, suppose $r$ is a CON of $x_{1,-1}$ and $x_{1, c}$ Let $q$ be a CON of $x_{2,-1}$ and $x_{1, c-1}$. Then the cycle

$$
u_{2} C\left[u_{2}, x_{1,-1}\right] x_{1,-1} r x_{1, c} C\left[x_{1, c}, x_{2,-1}\right] x_{2,-1} q x_{1, c-1} C^{-}\left[x_{1, c-1}, u_{1}\right] u_{1} F\left[u_{1}, u_{2}\right] u_{2}
$$

shown on the right of Figure 13 is longer than $C$.

Therefore we have a good set of size 4, a contradiction to Lemma 3.1. This proves (7). In other words, $x_{1,1}, x_{1,2}, \ldots, x_{1, b-1}$ all have a CON with $x_{1,-1}$ while $x_{1, b}, x_{1, b+1}, \ldots, x_{2,-2}$ all have a CON 

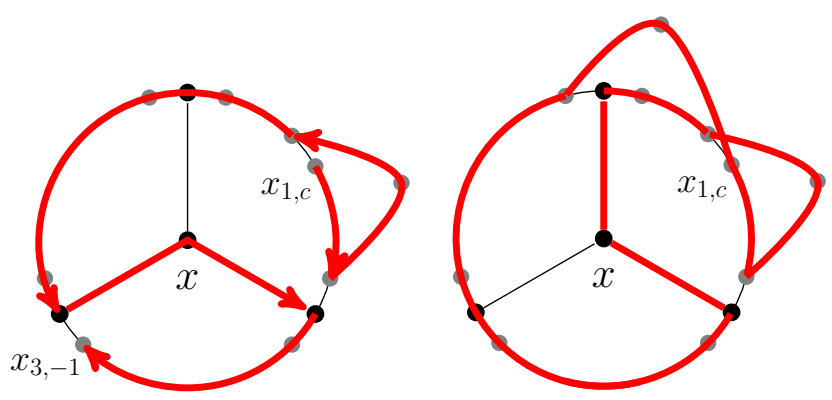

Figure 13: An $x_{1, c}, x_{3,-1}$-path, and a longer cycle obtained when $x_{1,-1}$ and $x_{1, c}$ have a CON.

with $x_{2,-1}$. Moreover, in this case, $x_{2,1}$ and $x_{2,-2}$ can have no CON, or else we obtain a longer cycle,

$x_{2,1} C\left[x_{2,1}, x_{1,-1}\right] x_{1,-1} r x_{1, b-1} C^{-}\left[x_{1, b-1}, u_{1}\right] u_{1} F\left[u_{1}, u_{2}\right] u_{2} C^{-}\left[u_{2}, x_{2,-1}\right] x_{2,-1} s x_{1, b} C\left[x_{1, b}, x_{2,-2}\right] x_{2,-2} t x_{2,1}$,

where $r$ is the CON of $x_{1,-1}$ and $x_{1, b-1}, s$ is the CON of $x_{2,-1}$ and $x_{1, b}$, and $t$ is the CON of $x_{2,-2}$ and $x_{2,1}$, as shown in Figure 14 .

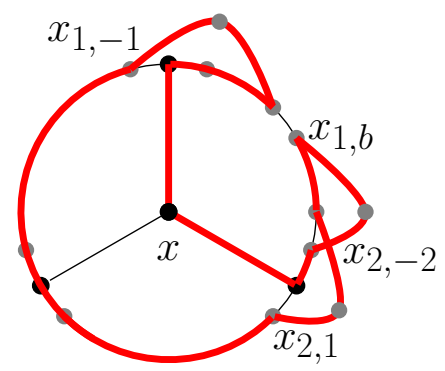

Figure 14: A longer cycle obtained when $x_{2,1}$ and $x_{2,-2}$ have a CON.

We can apply the argument in this subsection in six possible ways: we can swap the roles of $x_{1,1}$ and $x_{1,-1}$ in the argument above, and we can choose any of the three short-type configurations in place of the one formed by $x_{1,1}$ and $x_{1,-1}$. All six of these arguments must terminate in the same case. In particular, just as we concluded that $x_{2,1}$ and $x_{2,-2}$ can have no CON, we also conclude that $x_{1,-1}$ and $x_{1,2}$ can have no CON. This means that in the argument above (and in all variations of the argument), we must have $b=2$.

Therefore, for each $i$, the vertices $x_{i, 2}, x_{i, 3}, \ldots, x_{i+1,-2}$ all have a CON with both $x_{i, 1}$ and with $x_{i+1,-1}$. In other words, all $i \in[3]$ are abundant.

By Lemma 4.10 and Lemma 4.11, no $i \in[3]$ has medium or long type. Therefore by Lemma 4.12 , every $i \in[3]$ is abundant. This contradicts Lemma 4.5, completing the proof of Theorem 1.4 . 


\section{Concluding remarks}

1. Theorem 1.4 is a natural 3-connected strengthening of Conjecture 1.1 for 2-connected graphs. Consider the following family of $k$-connected graphs.

Construction 5.1. Let $k$ be a positive integer, and let $n_{1} \geq \ldots \geq n_{k+1} \geq 1$ be such that $n_{1}+\ldots+n_{k+1}=n$. Let $G_{k}\left(n_{1}, \ldots, n_{k+1} ; \delta\right) \in \mathcal{G}(n,(k+1)(\delta-k)+k, \delta)$ be the bipartite graph obtained from $K_{\delta-k, n_{1}} \cup \ldots \cup K_{\delta-k, n_{k+1}}$ by adding $k$ vertices $a_{1}, \ldots, a_{k}$ that are each adjacent to every vertex in the parts of size $n_{1}, \ldots, n_{k+1}$. Let $\mathcal{G}_{k}(n, \delta)$ be the collection of the graphs $G_{k}\left(n_{1}, \ldots, n_{k+1} ; \delta\right)$ for all suitable choices of $n_{1}, \ldots, n_{k+1}$.

When $k=2$ or $k=3, \mathcal{G}_{k}$ is the family of all graphs in Construction 1.2 or Construction 1.3 respectively.

Question 5.2. Let $m, n, k, \delta$ be integers. Suppose $k \geq 4, \delta \geq n$ and $m \leq(k+1)(\delta-k)+$ $k-1$. Is it true that every $k$-connected graph $G \in \mathcal{G}(n, m, \delta)$ contains a cycle of length $2 n$ ? Moreover, if $k \geq 3$, are the graphs in the family $\mathcal{G}_{k}(n, \delta)$ the only extremal examples with $m=(k+1)(\delta-k)+k$ ?

If the answer is negative, it would also be interesting to find the value(s) of $k$ at which other extremal examples occur.

2. Jackson also made the following conjecture.

Conjecture 5.3 (Jackson [3]). Let $m, n, \delta$ be integers with $n>\delta$. If a graph $G \in \mathcal{G}(n, m, \delta)$ is 2-connected and satisfies

$$
m \leq\left\lfloor\frac{2(n-\alpha)}{\delta-1-\alpha}\right\rfloor(\delta-2)+1
$$

where $\alpha=1$ if $\delta$ is even and $\alpha=0$ if $\delta$ is odd, then $G$ contains a cycle of length at least $2 \min (n, \delta)$.

This conjecture remains open. A weaker version is proved in [6] in the language of hypergraphs.

\section{References}

[1] J. A. Bondy, Pancyclic graphs I, J. Combin. Theory 11, (1971), 80-84.

[2] J. A. Bondy, Pancyclic graphs: recent results, Infinite and finite sets (Colloq., Keszthely, 1973; dedicated to P. Erdős on his 60th birthday), Vol. I, 181-187. Colloq. Math. Soc. János Bolyai, Vol. 10, North-Holland, Amsterdam (1975).

[3] B. Jackson, Cycles in bipartite graphs, J. Combin. Theory, Ser. B, 30 (1981), 332-342.

[4] B. Jackson, Maximal cycles in bipartite graphs, Combinatorial Mathematics, Vol. 75, NorthHolland, Amsterdam (1983), 361-363.

[5] B. Jackson, Long cycles in bipartite graphs, J. Combin. Theory, Ser. B, 38 (1985), 118-131.

[6] A. Kostochka, and R. Luo, On $r$-uniform hypergraphs with circumference less than $r$, Discrete Appl. Math., 276 (2020), 69-91. 
[7] A. Kostochka, R. Luo, and D. Zirlin, Super-pancyclic hypergraphs and bipartite graphs, submitted, https://arxiv.org/pdf/1905.03758.pdf, 13 pp.

[8] J. Mitchem and E. Schmeichel, Pancyclic and bipancyclic graphs - a survey, Graphs and applications (1985), 271-278. 